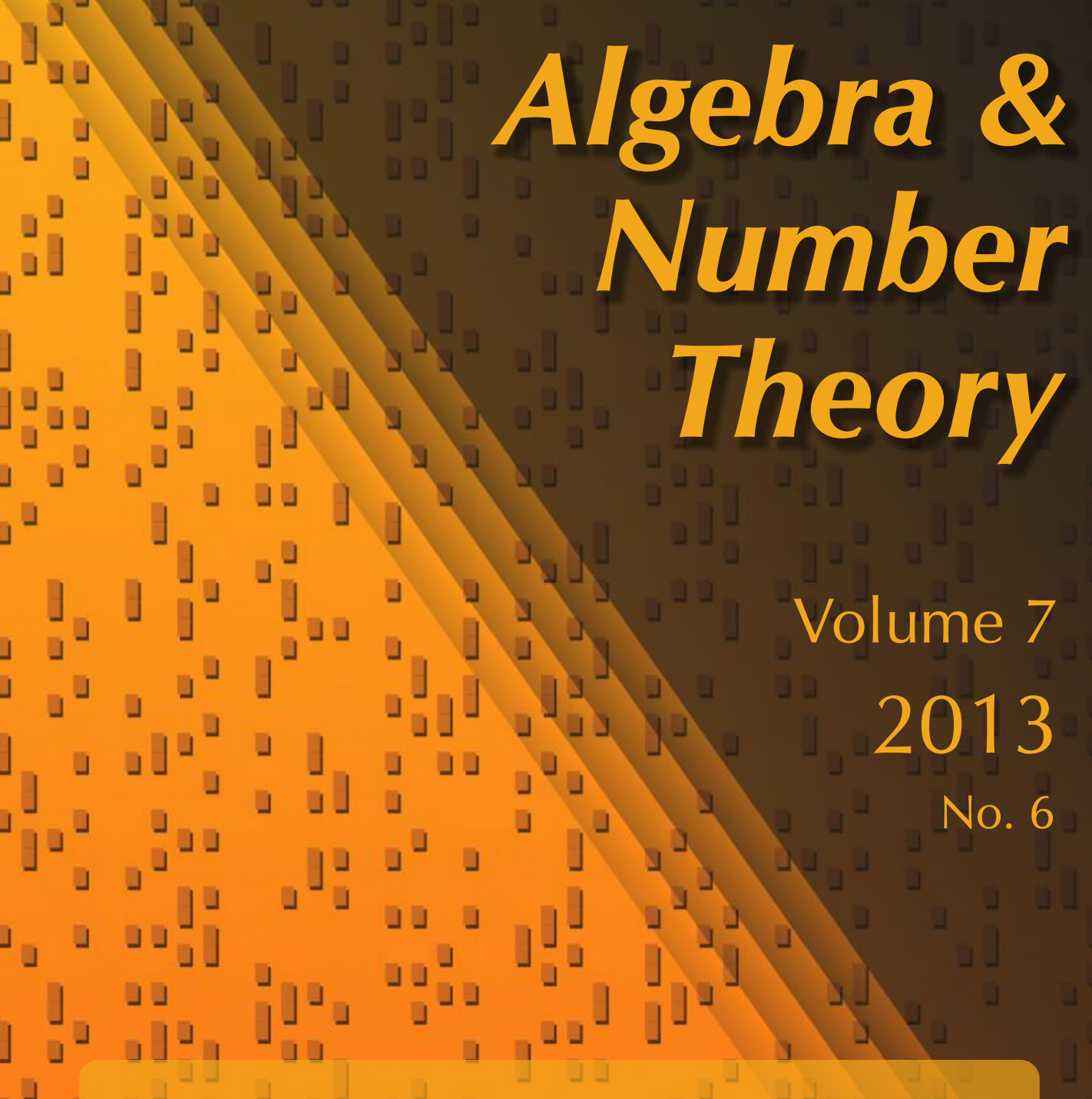

Commuting involutions of Lie algebras, commuting varieties, and simple Jordan algebras

Dmitri I. Panyushev

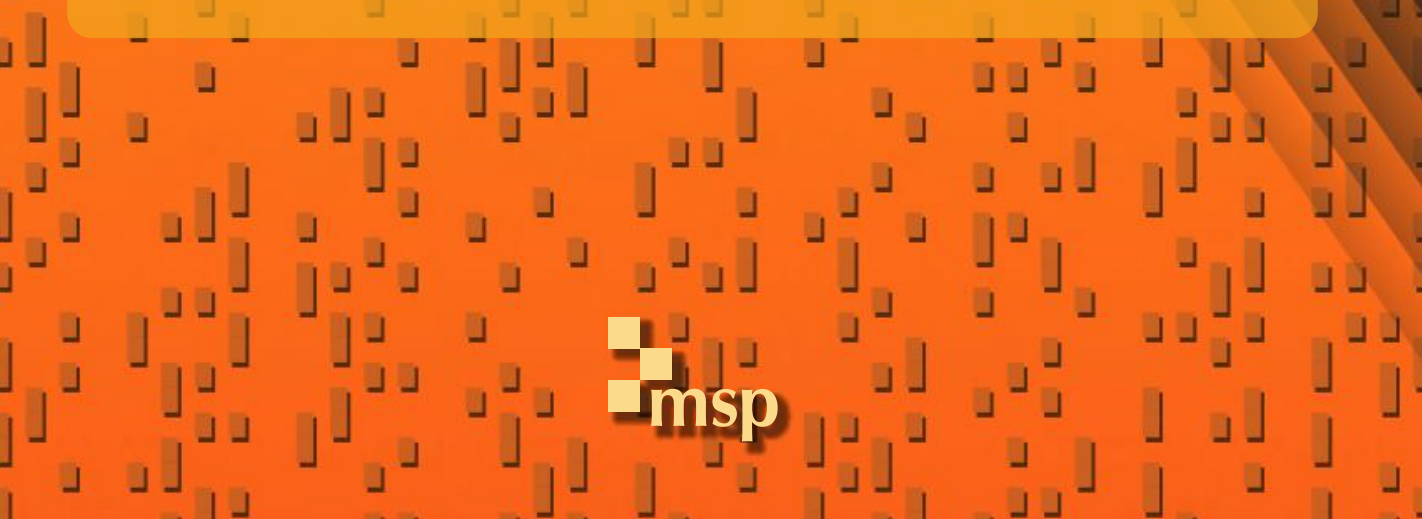




\title{
Commuting involutions of Lie algebras, commuting varieties, and simple Jordan algebras
}

\author{
Dmitri I. Panyushev
}

Let $\sigma_{1}$ and $\sigma_{2}$ be commuting involutions of a connected reductive algebraic group $G$ with $\mathfrak{g}=\operatorname{Lie}(G)$. Let

$$
\mathfrak{g}=\bigoplus_{i, j=0,1} \mathfrak{g}_{i j}
$$

be the corresponding $\mathbb{Z}_{2} \times \mathbb{Z}_{2}$-grading. If $\{\alpha, \beta, \gamma\}=\{01,10,11\}$, then [ , ] maps $\mathfrak{g}_{\alpha} \times \mathfrak{g}_{\beta}$ into $\mathfrak{g}_{\gamma}$, and the zero fiber of this bracket is called a $\overrightarrow{\boldsymbol{\sigma}}$-commuting variety. The commuting variety of $\mathfrak{g}$ and commuting varieties related to one involution are particular cases of this construction. We develop a general theory of such varieties and point out some cases, when they have especially good properties. If $G / G^{\sigma_{1}}$ is a Hermitian symmetric space of tube type, then one can find three conjugate pairwise commuting involutions $\sigma_{1}, \sigma_{2}$, and $\sigma_{3}=\sigma_{1} \sigma_{2}$. In this case, any $\overrightarrow{\boldsymbol{\sigma}}$-commuting variety is isomorphic to the commuting variety of the simple Jordan algebra associated with $\sigma_{1}$. As an application, we show that if $\mathcal{J}$ is the Jordan algebra of symmetric matrices, then the product map $\mathcal{J} \times \mathcal{J} \rightarrow \mathcal{J}$ is equidimensional, while for all other simple Jordan algebras equidimensionality fails.

Introduction

1. Preliminaries on involutions and commuting varieties

2. Commuting involutions and quaternionic decompositions

3. Commuting varieties and homogeneous Cartan subspaces

4. Dyads of maximal rank and commuting varieties

5. Commuting varieties and restricted root systems

6. Triads of Hermitian involutions and simple Jordan algebras

Appendix: Computations in classical Lie algebras

References

MSC2010: primary 14L30; secondary 17B08, 17B40, 17C20, 22E46.

Keywords: semisimple Lie algebra, commuting variety, Cartan subspace, quaternionic decomposition, nilpotent orbit, Jordan algebra. 


\section{Introduction}

The ground field $\mathbb{k}$ is algebraically closed and char $\mathbb{k}=0$. Let $G$ be a connected reductive algebraic group with $\operatorname{Lie}(G)=\mathfrak{g}$. Richardson [1979] proved that any pair of commuting elements of $\mathfrak{g}$ can be approximated by pairs of commuting semisimple elements. More precisely, if $\mathfrak{t} \subset \mathfrak{g}$ is a Cartan subalgebra (CSA), then

$$
\{(x, y) \in \mathfrak{g} \times \mathfrak{g} \mid[x, y]=0\}=\overline{G \cdot(\mathfrak{t} \times \mathfrak{t})},
$$

where a bar indicates the Zariski closure. The left-hand side is called the commuting variety of $\mathfrak{g}$, denoted $\mathfrak{E}(\mathfrak{g})$. That is, $\mathfrak{E}(\mathfrak{g})$ is the zero fiber of the multiplication map

$$
\mathfrak{g} \times \mathfrak{g} \stackrel{[,]}{\longrightarrow} \mathfrak{g} .
$$

It follows from $(0-1)$ that $\mathfrak{E}(\mathfrak{g})$ is irreducible and $\operatorname{dim} \mathfrak{E}(\mathfrak{g})=\operatorname{dim} \mathfrak{g}+r \mathfrak{r k}$. For arbitrary Lie algebras, for example, for Borel subalgebras of $\mathfrak{g}$, the commuting variety can be reducible [Vasconcelos 1994, p. 237].

There are several directions to take in generalizing Richardson's work.

First, for given subvarieties $U, V \subset \mathfrak{g}$, one can consider the restriction of [ , ] to $U \times V$ and study properties of $\mathfrak{E}(\mathfrak{g}) \cap(U \times V)$. For instance:

- Let $\sigma$ be an involution of $\mathfrak{g}$ with the corresponding $\mathbb{Z}_{2}$-grading $\mathfrak{g}=\mathfrak{g}_{0} \oplus \mathfrak{g}_{1}$. Taking $U=V=\mathfrak{g}_{1}$ yields the commuting variety $\mathfrak{E}\left(\mathfrak{g}_{1}\right):=\mathfrak{E}(\mathfrak{g}) \cap\left(\mathfrak{g}_{1} \times \mathfrak{g}_{1}\right)$, which was considered first in [Panyushev 1994b]. Here the structure of $\mathfrak{E}\left(\mathfrak{g}_{1}\right)$ heavily depends on $\sigma$. If $\mathfrak{g}_{1}$ contains a CSA of $\mathfrak{g}$, then $\mathfrak{E}\left(\mathfrak{g}_{1}\right)$ is an irreducible normal complete intersection [Panyushev 1994b]. At the other extreme, if the symmetric space $G / G_{0}$ is of rank 1 , then $\mathfrak{E}\left(\mathfrak{g}_{1}\right)$ is often reducible. In [Panyushev and Yakimova 2007], the question of irreducibility of $\mathfrak{E}\left(\mathfrak{g}_{1}\right)$ is resolved for all but three involutions of simple Lie algebras, and the remaining cases are settled in [Bulois 2011]. It seems, however, that there is no simple rule to distinguish the involutions for which $\mathfrak{E}\left(\mathfrak{g}_{1}\right)$ is irreducible.

- Another natural possibility is to take $U=V=\mathcal{N}$, where $\mathcal{N}$ is the set of nilpotent elements of $\mathfrak{g}$. This leads to the nilpotent commuting variety of $\mathfrak{g}, \mathfrak{E}(\mathcal{N})$, which is often reducible. However, $\mathfrak{E}(\mathcal{N})$ is equidimensional, $\operatorname{dim} \mathfrak{E}(\mathcal{N})=\operatorname{dim} \mathfrak{g}$, and the structure of irreducible components is well understood [Premet 2003].

- An interesting situation with $U \neq V$ occurs if $\mathfrak{g}=\bigoplus_{i \in \mathbb{Z}} \mathfrak{g}(i)$ is $\mathbb{Z}$-graded, $U=\mathfrak{g}(i)$, and $V=\mathfrak{g}(-i)$, see [Panyushev 1999, §3].

Second, one may look at commuting varieties related to other types of algebras. If $\mathcal{A}$ is any algebra, then $\mathfrak{E}(\mathcal{A})$ is defined to be the zero fiber of the multiplication map $\mathcal{A} \times \mathcal{A} \rightarrow \mathcal{A}$. It is a natural task to study the commuting variety of a simple Jordan algebra. As far as I know, this problem has not been addressed before. 
In this article, we elaborate on both directions outlined above. We study certain "commuting varieties" associated with $\mathbb{Z}_{2} \times \mathbb{Z}_{2}$-gradings of $\mathfrak{g}$ (the first direction). It turns out that, for some gradings, these new commuting varieties are isomorphic to the commuting variety of simple Jordan algebras (the second direction). To describe our results more precisely, we need some notation. Let $\sigma_{1}$ and $\sigma_{2}$ be different commuting involutions of a connected reductive algebraic group $G$. This yields a $\mathbb{Z}_{2} \times \mathbb{Z}_{2}$-grading of $\mathfrak{g}$ :

$$
\mathfrak{g}=\bigoplus_{i, j=0,1} \mathfrak{g}_{i j}, \text { where } \mathfrak{g}_{i j}=\left\{x \in \mathfrak{g} \mid \sigma_{1}(x)=(-1)^{i} x \text { and } \sigma_{2}(x)=(-1)^{j} x\right\}
$$

Then $\sigma_{1}, \sigma_{2}$, and $\sigma_{3}=\sigma_{1} \sigma_{2}$ are pairwise commuting involutions, and following [Vergne 1995] we say that (0-2) is a quaternionic decomposition of $\mathfrak{g}$. For, if $(\alpha, \beta, \gamma)$ is any permutation of the set of indices $\{01,10,11\}$, then $\left[\mathfrak{g}_{00}, \mathfrak{g}_{\alpha}\right] \subset \mathfrak{g}_{\alpha}$ and $\left[\mathfrak{g}_{\alpha}, \mathfrak{g}_{\beta}\right] \subset \mathfrak{g}_{\gamma}$. The conjugacy classes of pairs of commuting involutions are classified, see [Kollross 2009] and references therein. Therefore, it is not difficult to write down explicitly all the quaternionic decompositions of simple Lie algebras. This article is a continuation of [Panyushev 2013], where we developed some theory on Cartan subspaces related to (0-2) and studied invariants of degenerations of isotropy representations involved.

Set $\overrightarrow{\boldsymbol{\sigma}}=\left(\sigma_{1}, \sigma_{2}, \sigma_{3}\right)$, and let $G_{00}$ denote the connected subgroup of $G$ with Lie algebra $\mathfrak{g}_{00}$. A $\overrightarrow{\boldsymbol{\sigma}}$-commuting variety is the zero fiber of the bracket $[$,$] :$ $\mathfrak{g}_{\alpha} \times \mathfrak{g}_{\beta} \rightarrow \mathfrak{g}_{\gamma}$. Associated with (0-2), one has three essentially different such varieties that are parameterized by the choice of $\gamma \in\{01,10,11\}$. All these mappings are $G_{00}$-equivariant, and all $\overrightarrow{\boldsymbol{\sigma}}$-commuting varieties are $G_{00}$-varieties. The abovementioned varieties $\mathfrak{E}\left(\mathfrak{g}_{1}\right)$ can be obtained as a special case of this construction, see Example 3.1. We usually stick to one particular choice of the commutator, $\varphi: \mathfrak{g}_{10} \times \mathfrak{g}_{11} \rightarrow \mathfrak{g}_{01}$, and try to realize what assumptions on $\overrightarrow{\boldsymbol{\sigma}}$ imply good properties of $\mathfrak{E}:=\varphi^{-1}(0)$ and other fibers of $\varphi$. Clearly, $\varphi$ can be regarded as a quadratic map from $\mathfrak{g}_{1 \star}:=\mathfrak{g}_{10} \oplus \mathfrak{g}_{11}$ to $\mathfrak{g}_{01}$. Let $\mathfrak{c}_{1 \star}$ be a Cartan subspace (CSS) in $\mathfrak{g}_{1 \star}$. Say that $\mathfrak{c}_{1 \star}$ is homogeneous if it is $\sigma_{2}$-stable (or, equivalently, $\sigma_{3}$-stable), that is, if $\mathfrak{c}_{1 \star}=\mathfrak{a}_{10} \oplus \mathfrak{a}_{11}$ with $\mathfrak{a}_{1 j} \subset \mathfrak{g}_{1 j}$. We prove:

- If $\mathfrak{c}_{1 \star}$ is a homogeneous CSS, then the closure of $G_{00} \cdot \mathfrak{c}_{1 \star}$ is an irreducible component of $\mathfrak{E}$ (Theorem 3.4). (Such irreducible components are said to be standard). However, there can be several standard components, of different dimensions, and there can also exist some "nonstandard" irreducible components.

- All homogeneous CSS in $\mathfrak{g}_{1 \star}$ are $G_{00}$-conjugate (that is, E has only one standard component) if and only if $\operatorname{dim} \mathfrak{c}_{1 \star}=\operatorname{dim} \mathfrak{c}_{10}+\operatorname{dim} \mathfrak{c}_{11}$, where $\mathfrak{c}_{1 j}$ are CSS in $\mathfrak{g}_{1 j}$ (Theorem 3.7). 
- The commutator map $\varphi$ is dominant if and only if there exist $x \in \mathfrak{g}_{10}$ and $y \in \mathfrak{g}_{11}$ such that $\mathfrak{z}_{\mathfrak{g}}(x)_{01} \cap \mathfrak{z}_{\mathfrak{g}}(y)_{01}=\{0\}$.

However, one cannot expect really good properties for $\varphi$ and $\mathfrak{E}$ without extra assumptions. One natural assumption is that some of involutions in $\vec{\sigma}$ are conjugate. Another possibility is that some of the $\sigma_{i}$ possess prescribed properties. Our more specific results are:

(1) If $\sigma_{1}$ and $\sigma_{2}$ are conjugate, then $\varphi$ is surjective and $\operatorname{dim} \varphi^{-1}(\xi) \geqslant \operatorname{dim} \mathfrak{g}_{11}$ for all $\xi \in \mathfrak{g}_{01}$ (Proposition 3.8). We also provide a method for detecting subvarieties of $\mathfrak{E}$ whose dimension is larger than $\operatorname{dim} \mathfrak{g}_{11}$. This exploits certain restricted root systems related to decomposition (0-2), see Section 5.

(2) If $\sigma_{1}$ and $\sigma_{2}$ are involutions of maximal rank (hence they are conjugate), then $\varphi$ is surjective and equidimensional, each irreducible component of $\mathfrak{E}$ is standard, and the scheme $\varphi^{-1}(0)$ is a reduced complete intersection (Theorem 4.1).

(3) Let $\mathfrak{g}$ be simple and $\sigma$ a Hermitian involution (that is, $\mathfrak{g}^{\sigma}$ is not semisimple). If the Hermitian symmetric space $G / G^{\sigma}$ is of tube type, then there exists a commuting triple $\overrightarrow{\boldsymbol{\sigma}}$ such that each $\sigma_{i}$ is conjugate to $\sigma$, and in this case $\mathfrak{E}$ is isomorphic to the commuting variety of the corresponding simple Jordan algebra, see Section 6.

(4) The relationship with $\overrightarrow{\boldsymbol{\sigma}}$-commuting varieties implies that the multiplication map $\mathcal{J} \times \mathcal{J} \stackrel{\circ}{\rightarrow} \mathcal{J}$ is equidimensional if and only if $\mathcal{J}$ is the Jordan algebra of symmetric matrices. The commuting variety of a simple Jordan algebra $\mathcal{J}$ is reducible, since $\mathcal{J} \times\{0\}$ and $\{0\} \times \mathcal{J}$ are always irreducible components, and there are certainly some other components.

(5) The results stated in (2) rely on an interesting property of $\mathbb{Z}_{2}$-gradings. For any $e \in \mathfrak{g}_{0}$, its centralizer in $\mathfrak{g}$ is also $\mathbb{Z}_{2}$-graded: $\mathfrak{g}^{e}=\mathfrak{g}_{0}^{e} \oplus \mathfrak{g}_{1}^{e}$. Then we prove that

$$
\operatorname{dim} \mathfrak{g}_{0}^{e}+\mathrm{rk} \mathfrak{g} \geqslant \operatorname{dim} \mathfrak{g}_{1}^{e}
$$

and the equality occurs only if $e=0$ and $\sigma$ is of maximal rank. However, the proof of this inequality (Theorem 4.4) is not quite uniform, and a better proof is welcome! The required case-by-case calculations are lengthy and tedious, so not all of them are actually presented, and a part is placed in the Appendix. We hope that an a priori proof of this inequality might be related to a geometric property of centralizers of nilpotent elements in $\mathfrak{g}_{0}$, see Conjecture 4.6.

- Throughout, $G$ is a connected reductive algebraic group and $\mathfrak{g}=\operatorname{Lie}(G)$. Then $\mathfrak{z}_{\mathfrak{g}}(\mathfrak{a})$ is the centralizer of a subspace $\mathfrak{a} \subset \mathfrak{g}$, and the centralizer of $x \in \mathfrak{g}$ is denoted by $\mathfrak{z}_{\mathfrak{g}}(x)$ or $\mathfrak{g}^{x}$.

- $\mathrm{R}(\lambda)$ is a simple finite-dimensional $G$-module with highest weight $\lambda$.

- Algebraic groups are denoted by capital Roman letters and their Lie algebras are denoted by the corresponding lower-case Gothic letters. 


\section{Preliminaries on involutions and commuting varieties}

The set of all involutions of $\mathfrak{g}$ is denoted by $\operatorname{Inv}(\mathfrak{g})$. The group of inner automorphisms $\operatorname{lnt}(G) \simeq G / Z(G)$ acts on $\operatorname{lnv}(\mathfrak{g})$ by conjugation. Two involutions are said to be conjugate if they lie in the same $\operatorname{Int}(G)$-orbit. If $\sigma \in \operatorname{Inv}(\mathfrak{g})$, then $\mathfrak{g}=\mathfrak{g}_{0} \oplus \mathfrak{g}_{1}$ is the corresponding $\mathbb{Z}_{2}$-grading of $\mathfrak{g}$, where $\mathfrak{g}_{i}=\left\{x \in \mathfrak{g} \mid \sigma(x)=(-1)^{i} x\right\}$. We also say that $\left(\mathfrak{g}, \mathfrak{g}_{0}\right)$ is a symmetric pair. Whenever we wish to stress that $\mathfrak{g}_{0}$ and $\mathfrak{g}_{1}$ are determined by $\sigma$, we write $\mathfrak{g}^{\sigma}$ and $\mathfrak{g}_{1}^{(\sigma)}$ for them. We assume that $\sigma$ is induced by an involution of $G$, which is denoted by the same letter. The connected subgroup of $G$ with Lie algebra $\mathfrak{g}_{0}$ is denoted by $G_{0}$. Hence $G_{0}$ is the identity component of $G^{\sigma}=\{g \in G \mid \sigma(g)=g\}$. The representation of $G_{0}$ in $\mathfrak{g}_{1}$ is the isotropy representation of the symmetric space $G / G_{0}$.

We freely use the invariant-theoretic results on the $G_{0}$-action on $\mathfrak{g}_{1}$ obtained in [Kostant and Rallis 1971]. A Cartan subspace (CSS) is a maximal subspace of $\mathfrak{g}_{1}$ consisting of pairwise commuting semisimple elements. The Cartan subspaces are characterized by the following property:

Suppose that a subspace $\mathfrak{a} \subset \mathfrak{g}_{1}$ consists of pairwise commuting semisim-

ple elements. Then $\mathfrak{a}$ is a CSS if and only if $\mathfrak{z}_{\mathfrak{g}}(\mathfrak{a}) \cap \mathfrak{g}_{1}=\mathfrak{a}$ [Kostant and

Rallis 1971, Chapter I].

An element $x \in \mathfrak{g}_{1}$ is called $G_{0}$-regular if the orbit $G_{0} \cdot x$ is of maximal dimension. Let $\mathfrak{c}$ be a CSS of $\mathfrak{g}_{1}$. Below, we summarize some basic properties of the Cartan subspaces and isotropy representations:

- All CSS of $\mathfrak{g}_{1}$ are $G_{0}$-conjugate and $G_{0} \cdot \mathfrak{c}$ is dense in $\mathfrak{g}_{1}$.

- Every semisimple element of $\mathfrak{g}_{1}$ is $G_{0}$-conjugate to an element of $\mathfrak{c}$.

- A semisimple element $x \in \mathfrak{g}_{1}$ is $G_{0}$-regular $\Longleftrightarrow \mathfrak{z}_{\mathfrak{g}}(x) \cap \mathfrak{g}_{1}$ is a CSS.

- The orbit $G_{0} \cdot x$ is closed if and only if $x$ is semisimple.

- The closure of $G_{0} \cdot x$ contains the origin if and only if $x$ is nilpotent.

- The number of nilpotent $G_{0}$-orbits in $\mathfrak{g}_{1}$ is finite.

We say that $\sigma \in \operatorname{lnv}(\mathfrak{g})$ is of maximal rank if $\mathfrak{g}_{1}$ contains a Cartan subalgebra of $\mathfrak{g}$.

The following facts are well known:

(1) $\operatorname{dim} \mathfrak{g}_{1}-\operatorname{dim} \mathfrak{g}_{0} \leqslant \mathrm{rkg}$ for any $\sigma$, and the equality holds if and only if $\sigma$ is of maximal rank.

(2) All involutions of maximal rank are conjugate.

(3) The involutions of maximal rank are inner if and only if all exponents of $\mathfrak{g}$ are odd. 
Lemma 1.1 [Kostant and Rallis 1971, Proposition 5]. For any $x \in \mathfrak{g}_{1}$, one has $\operatorname{dim} \mathfrak{g}_{0}-\operatorname{dim} \mathfrak{g}_{0}^{x}=\operatorname{dim} \mathfrak{g}_{1}-\operatorname{dim} \mathfrak{g}_{1}^{x}$. Equivalently, $\operatorname{dim} G \cdot x=2 \operatorname{dim} G_{0} \cdot x$ for all $x \in \mathfrak{g}_{1}$.

Consequently, if $\sigma$ is of maximal rank, then

$$
\operatorname{dim} \mathfrak{g}_{1}^{x}=\operatorname{dim} \mathfrak{g}_{0}^{x}+\text { rk } \mathfrak{g} .
$$

The property of having maximal rank is inheritable in the following sense.

Lemma 1.2. Let $\sigma$ be of maximal rank and $x \in \mathfrak{g}_{1}$ semisimple. Then the restriction of $\sigma$ to $\mathfrak{g}^{x}$ and $\left[\mathfrak{g}^{x}, \mathfrak{g}^{x}\right]$ is also of maximal rank.

The commuting variety associated with $\sigma$ is

$$
\mathfrak{E}\left(\mathfrak{g}_{1}\right)=\left\{(x, y) \in \mathfrak{g}_{1} \times \mathfrak{g}_{1} \mid[x, y]=0\right\} .
$$

That is, $\mathfrak{E}\left(\mathfrak{g}_{1}\right)$ is the zero fiber of the commutator map $[,]_{1}: \mathfrak{g}_{1} \times \mathfrak{g}_{1} \rightarrow \mathfrak{g}_{0}$. The following is known:

- $\overline{G_{0} \cdot(\mathfrak{c} \times \mathfrak{c})}$ is always an irreducible component of $\mathfrak{E}\left(\mathfrak{g}_{1}\right)$ [Panyushev 1994b, Proposition 3.7].

- If $\sigma$ is of maximal rank, then $\overline{G_{0} \cdot(\mathfrak{c} \times \mathfrak{c})}=\mathfrak{E}\left(\mathfrak{g}_{1}\right)$ and $\mathfrak{g}_{1} \times \mathfrak{g}_{1} \rightarrow \mathfrak{g}_{0}$ is equidimensional [Panyushev 1994b, Theorem 3.2]; moreover, all the fibers of [ , ]1 are irreducible and normal [Panyushev 1994b, Corollary 4.4].

- $\mathfrak{E}\left(\mathfrak{g}_{1}\right)$ can be reducible [Panyushev 1994b, Example 3.5].

Example 1.3. Suppose that $\tilde{\mathfrak{g}}=\mathfrak{g} \oplus \mathfrak{g}$ and $\sigma(x, y)=(y, x)$. Then $\tilde{\mathfrak{g}}_{0}=\Delta(\mathfrak{g})$ and $\tilde{\mathfrak{g}}_{1}=\{(x,-x) \mid x \in \mathfrak{g}\}$. Here the commutator $\tilde{\mathfrak{g}}_{1} \times \tilde{\mathfrak{g}}_{1} \rightarrow \tilde{\mathfrak{g}}_{0}$ coincides with the usual commutator $\mathfrak{g} \times \mathfrak{g} \rightarrow \mathfrak{g}$ and $\mathfrak{E}\left(\tilde{\mathfrak{g}}_{1}\right)$ is isomorphic to the usual commuting variety of a semisimple Lie algebra $\mathfrak{g}$. By a result of Richardson [1979], $\mathfrak{E}(\mathfrak{g})$ is irreducible and $\operatorname{dim} \mathfrak{E}(\mathfrak{g})=\operatorname{dim} \mathfrak{g}+$ rk $\mathfrak{g}$.

A torus $S$ of $G$ is called $\sigma$-anisotropic if $\sigma(s)=s^{-1}$ for all $s \in S$. All maximal $\sigma$-anisotropic tori are $G_{0}$-conjugate, and if $C \subset G$ is a maximal $\sigma$-anisotropic torus, then $\operatorname{Lie}(C)$ is a CSS in $\mathfrak{g}_{1}$. Recall that a restricted root of $C$ is any nontrivial weight in the decomposition of $\mathfrak{g}$ into the sum of weight spaces of $C$. Write $\Psi^{C}\left(G / G_{0}\right)$ or just $\Psi\left(G / G_{0}\right)$ for the set of all restricted roots. Then

$$
\mathfrak{g}=\mathfrak{g}^{C} \oplus\left(\bigoplus_{\gamma \in \Psi\left(G / G_{0}\right)} \mathfrak{g}_{\gamma}\right) .
$$

We use additive notation for the operation in $\mathfrak{X}(C)$, the character group of $C$, and regard $\Psi\left(G / G_{0}\right)$ as a subset of the vector space $\mathfrak{X}(C) \otimes_{\mathbb{Z}} \mathbb{R}$. The set $\Psi\left(G / G_{0}\right)$ satisfies the usual axioms of finite root systems [Helgason 1978]. The notable difference from the structure theory of split semisimple Lie algebras is that the 
root system $\Psi\left(G / G_{0}\right)$ can be nonreduced and that multiplicities $m_{\gamma}=\operatorname{dim} \mathfrak{g}_{\gamma}$ $\left(\gamma \in \Psi\left(G / G_{0}\right)\right)$ can be greater than 1 .

For all involutions of simple Lie algebras, the restricted root systems and the respective multiplicities are known, see [Helgason 1978, Chapter X, Table VI].

\section{Commuting involutions and quaternionic decompositions}

Let $\sigma_{1}$ and $\sigma_{2}$ be different commuting involutions of $\mathfrak{g}$. Then the corresponding $\mathbb{Z}_{2} \times \mathbb{Z}_{2}$-grading of $\mathfrak{g}$ is

$$
\mathfrak{g}=\bigoplus_{i, j=0,1} \mathfrak{g}_{i j}, \text { where } \mathfrak{g}_{i j}=\left\{x \in \mathfrak{g} \mid \sigma_{1}(x)=(-1)^{i} x \text { and } \sigma_{2}(x)=(-1)^{j} x\right\}
$$

We also say that it is a quaternionic decomposition of $\mathfrak{g}$ (determined by $\sigma_{1}$ and $\sigma_{2}$ ). Set $\sigma_{3}:=\sigma_{1} \sigma_{2}$ and $\overrightarrow{\boldsymbol{\sigma}}=\left(\sigma_{1}, \sigma_{2}, \sigma_{3}\right)$. The pairwise commuting involutions $\sigma_{1}, \sigma_{2}$, and $\sigma_{3}$ are said to be big. The induced involutions on the fixed-point subalgebras $\mathfrak{g}^{\sigma_{1}}$, $\mathfrak{g}^{\sigma_{2}}$, and $\mathfrak{g}^{\sigma_{3}}$ are said to be little. The same terminology applies to the corresponding $\mathbb{Z}_{2}$-gradings, isotropy representations, and CSS. Thus, associated with (2-1), one has three big and three little $\mathbb{Z}_{2}$-gradings. It is convenient for us to organize the summands of (2-1) in a $2 \times 2$ "matrix":

$$
\mathfrak{g}=\stackrel{\mathfrak{g}_{00}}{\mathfrak{g}_{10}} \underset{\sigma_{2}}{\oplus} \underset{\mathfrak{g}_{11}}{\mathfrak{g}_{01}} \sigma_{1}
$$

Here the horizontal (resp. vertical) dotted line separates the eigenspaces of $\sigma_{1}$ (resp. $\sigma_{2}$ ), whereas two diagonals of this matrix represent the eigenspaces of $\sigma_{3}$. Hence the first row, first column, and main diagonal represent the three little $\mathbb{Z}_{2}$-gradings (of $\mathfrak{g}^{\sigma_{1}}, \mathfrak{g}^{\sigma_{2}}$, and $\mathfrak{g}^{\sigma_{3}}$, respectively).

We repeatedly use the following notation for the eigenspaces of $\sigma_{1}$ and $\sigma_{2}$ :

$\mathfrak{g}^{\sigma_{1}}=\mathfrak{g}_{0 \star}:=\mathfrak{g}_{00} \oplus \mathfrak{g}_{01}, \mathfrak{g}_{1 \star}:=\mathfrak{g}_{10} \oplus \mathfrak{g}_{11}, \quad \mathfrak{g}^{\sigma_{2}}=\mathfrak{g}_{\star 0}:=\mathfrak{g}_{00} \oplus \mathfrak{g}_{10}, \mathfrak{g}_{\star 1}:=\mathfrak{g}_{01} \oplus \mathfrak{g}_{11}$.

Likewise, $G_{0 \star}$ (resp. $G_{\star 0}$ ) is the connected subgroup of $G$ corresponding to $\mathfrak{g}_{0 \star}$ (resp. $\left.\mathfrak{g}_{\star 0}\right), G_{00}$ is the connected subgroup of $G$ corresponding to $\mathfrak{g}_{00}$, etc. If $\mathfrak{q}$ is a $\overrightarrow{\boldsymbol{\sigma}}$-stable subalgebra of $\mathfrak{g}$, then $\mathfrak{q}=\bigoplus_{i, j} \mathfrak{q}_{i j}$ is the induced quaternionic decomposition of $\mathfrak{q}$, and $Q$ and $Q_{00}$ are the corresponding connected subgroups.

Following [Vinberg 2005, 0.3], we say that a triple $\left\{\sigma_{1}, \sigma_{2}, \sigma_{3}\right\} \subset \operatorname{lnv}(\mathfrak{g})$ is a triad if all three involutions are conjugate and $\sigma_{1} \sigma_{2}=\sigma_{3}$. A complete classification of triads is obtained in [Vinberg 2005, §3]. The triads lead to the "most symmetric" quaternionic decompositions. In [Panyushev 2013], we considered less restrictive conditions on the $\sigma_{i}$. We say that $\left\{\sigma_{1}, \sigma_{2}\right\} \subset \operatorname{lnv}(\mathfrak{g})$ is a dyad if $\sigma_{1}$ and $\sigma_{2}$ are conjugate and $\sigma_{1} \sigma_{2}=\sigma_{2} \sigma_{1}$ (no conditions on $\sigma_{3}$ !). 
The product of two conjugate involutions (not necessarily commuting) is always an inner automorphism of $\mathfrak{g}$. For, if $\sigma_{2}=\operatorname{Int}(g) \cdot \sigma_{1} \cdot \operatorname{Int}\left(g^{-1}\right)$, then $\sigma_{1} \sigma_{2}=$ $\operatorname{Int}\left(\sigma_{1}(g) g^{-1}\right)$. Therefore, any triad consists of inner involutions. (But not every inner involution gives rise to a triad!) Any involution can be a member of a dyad [Panyushev 2013, Proposition 2.4]. But the third involution, $\sigma_{3}$, is then necessarily inner.

Proposition 2.1 (see [Panyushev 2013, Proposition 2.2(1)]). Suppose that $\mu \in$ $\operatorname{Inv}(\mathfrak{g})$ is inner. Then there are commuting involutions of maximal rank, $\sigma_{1}$ and $\sigma_{2}$, such that $\mu=\sigma_{1} \sigma_{2}$. Moreover, $\sigma_{1}$ and $\sigma_{2}$ induce an involution of maximal rank of $\mathfrak{g}^{\mu}$.

For $(i j) \neq(00)$, let $\mathfrak{c}_{i j}$ be a CSS of $\mathfrak{g}_{i j}$; that is, a little CSS related to the little $\mathbb{Z}_{2}$-grading $\mathfrak{g}_{00} \oplus \mathfrak{g}_{i j}$. There are also big CSS in the (-1)-eigenspaces of three big involutions:

$$
\mathfrak{c}_{1 \star} \subset \mathfrak{g}_{1 \star}, \quad \mathfrak{c}_{\star 1} \subset \mathfrak{g}_{\star 1}, \quad \mathfrak{c}_{\star, 1-\star} \subset \mathfrak{g}_{\star, 1-\star}:=\mathfrak{g}_{01} \oplus \mathfrak{g}_{10} .
$$

Each little CSS can be included in two big CSS. For example, because $\mathfrak{g}_{10} \subset \mathfrak{g}_{1 \star}$ and $\mathfrak{g}_{10} \subset \mathfrak{g}_{\star, 1-\star}$, one can choose Cartan subspaces $\mathfrak{c}_{1 \star}$ and $\mathfrak{c}_{\star, 1-\star}$ such that $\mathfrak{c}_{10} \subset \mathfrak{c}_{1 \star}$ and $\mathfrak{c}_{10} \subset \mathfrak{c}_{\star, 1-\star}$. If at least one equality occurs among all such inclusions, then this will be referred to as a coincidence of CSS (for a given quaternionic decomposition).

In [Panyushev 2013], we obtained two sufficient conditions for a coincidence of CSS:

Theorem 2.2 (see [Panyushev 2013, Theorems 3.3 and 3.7]).

(1) Suppose that $\sigma_{1}$ is of maximal rank. Then

- any little CSS $\mathfrak{c}_{11} \subset \mathfrak{g}_{11}$ is also a CSS in $\mathfrak{g}_{\star 1}$, that is, for $\sigma_{2}$, and

- any little CSS $\mathfrak{c}_{10} \subset \mathfrak{g}_{10}$ is also a CSS in $\mathfrak{g}_{10} \oplus \mathfrak{g}_{01}$, that is, for $\sigma_{3}$.

(2) Suppose that $\left\{\sigma_{1}, \sigma_{2}\right\}$ is a dyad. Then any little CSS $\mathfrak{c}_{11} \subset \mathfrak{g}_{11}$ is also a CSS in $\mathfrak{g}_{1 \star}$ or $\mathfrak{g}_{\star 1}$, that is, for $\sigma_{1}$ or $\sigma_{2}$.

The coincidences of CSS in Theorem 2.2(2) can formally be expressed as $\mathfrak{c}_{11}=\mathfrak{c}_{1 \star}$ or $\mathfrak{c}_{11}=\mathfrak{c}_{\star 1}$, and likewise in all other possible cases. In view of (1-1), any coincidence of CSS can be restated as certain property of the little CSS in question. For instance, the first coincidence in Theorem 2.2(1) means that if $x \in \mathfrak{g}_{11}$ is a generic semisimple element (that is, $x$ belong to a unique little CSS), then $\mathfrak{z}_{\mathfrak{g}}(x)_{\star 1}=\mathfrak{z}_{\mathfrak{g}}(x)_{11}=\mathfrak{c}_{11}$, and hence $\mathfrak{z} \mathfrak{g}(x)_{01}=0$.

\section{Commuting varieties and homogeneous Cartan subspaces}

Consider a quaternionic decomposition (2-2). For any permutation $(\alpha, \beta, \gamma)$ of the set $\{01,10,11\}$, there is the commutator mapping $\varphi_{\alpha, \beta}^{\gamma}: \mathfrak{g}_{\alpha} \times \mathfrak{g}_{\beta} \rightarrow \mathfrak{g}_{\gamma}$. Clearly, 
$\varphi_{\alpha, \beta}^{\gamma}$ is $G_{00}$-equivariant. As our main interest is in fibers of this mapping, we do not distinguish $\varphi_{\alpha, \beta}^{\gamma}$ and $\varphi_{\beta, \alpha}^{\gamma}$. We concentrate on the following problems:

- When is $\varphi_{\alpha, \beta}^{\gamma}$ dominant?

- What is the dimension of $\left(\varphi_{\alpha, \beta}^{\gamma}\right)^{-1}(0)$ ?

- How to describe the irreducible components of $\left(\varphi_{\alpha, \beta}^{\gamma}\right)^{-1}(0)$ ?

- When is $\varphi_{\alpha, \beta}^{\gamma}$ equidimensional?

The variety $\mathfrak{E}_{\alpha, \beta}^{\gamma}=\left(\varphi_{\alpha, \beta}^{\gamma}\right)^{-1}(0)$ is said to be a $\overrightarrow{\boldsymbol{\sigma}}$-commuting variety. For general quaternionic decompositions, one has three such varieties, and their properties can be rather different. We mainly restrict ourselves to considering the test case:

$$
\varphi=\varphi_{10,11}^{01}: \mathfrak{g}_{10} \times \mathfrak{g}_{11} \rightarrow \mathfrak{g}_{01} .
$$

and also write $\mathfrak{E}$ in place of $\mathfrak{E}_{10,11}^{01}$. Note that we can regard $\varphi$ as a quadratic map from $\mathfrak{g}_{1 \star}$ to $\mathfrak{g}_{01}$, and $\mathfrak{E}$ as subvariety of $\mathfrak{g}_{1 \star}$. The following example shows that the commuting variety in (1-3) is a particular case of this construction.

Example 3.1. Let $\mathfrak{g}$ be a reductive Lie algebra and $\sigma$ an involution of $\mathfrak{g}$ with the corresponding $\mathbb{Z}_{2}$-grading $\mathfrak{g}=\mathfrak{g}_{0} \oplus \mathfrak{g}_{1}$. Set $\tilde{\mathfrak{g}}=\mathfrak{g} \oplus \mathfrak{g}$ and define three involutions of $\tilde{\mathfrak{g}}$ as follows:

$$
\sigma_{1}\left(x_{1}, x_{2}\right)=\left(\sigma\left(x_{1}\right), \sigma\left(x_{2}\right)\right), \quad \sigma_{2}\left(x_{1}, x_{2}\right)=\left(x_{2}, x_{1}\right), \quad \sigma_{3}=\sigma_{1} \sigma_{2} .
$$

Then $\tilde{\mathfrak{g}}^{\sigma_{1}}=\mathfrak{g}_{0} \oplus \mathfrak{g}_{0} ; \tilde{\mathfrak{g}}^{\sigma_{2}}=\Delta(\mathfrak{g})$, the diagonal in $\mathfrak{g} \oplus \mathfrak{g} ;$ and $\tilde{\mathfrak{g}}^{\sigma_{3}}=\{(x, \sigma(x)) \mid x \in \mathfrak{g}\}$. Set $\Delta_{-}(M):=\{(m,-m) \mid m \in M\}$ for any subspace $M \subset \mathfrak{g}$. Then the corresponding quaternionic decomposition is

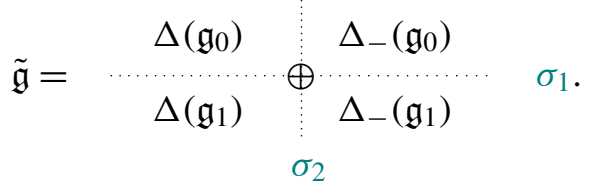

Upon the obvious identifications $\Delta\left(\mathfrak{g}_{1}\right) \simeq \Delta_{-}\left(\mathfrak{g}_{1}\right) \simeq \mathfrak{g}_{1}$, etc., our test commutator map $\tilde{\mathfrak{g}}_{10} \times \tilde{\mathfrak{g}}_{11} \rightarrow \tilde{\mathfrak{g}}_{01}$ becomes the commutator $\mathfrak{g}_{1} \times \mathfrak{g}_{1} \rightarrow \mathfrak{g}_{0}$ associated with $\sigma \in \operatorname{lnv}(\mathfrak{g})$; whereas two other commutator maps are identified with the bracket $\mathfrak{g}_{0} \times \mathfrak{g}_{1} \rightarrow \mathfrak{g}_{1}$. Therefore, the concept of a $\overrightarrow{\boldsymbol{\sigma}}$-commuting variety provides a uniform setting for studying the fibers of both $\mathfrak{g}_{1} \times \mathfrak{g}_{1} \rightarrow \mathfrak{g}_{0}$ and $\mathfrak{g}_{0} \times \mathfrak{g}_{1} \rightarrow \mathfrak{g}_{1}$.

Lemma 3.2. The commutator map (3-1) is dominant if and only if there exist $x \in \mathfrak{g}_{10}$ and $y \in \mathfrak{g}_{11}$ such that $\mathfrak{z}_{\mathfrak{g}}(x)_{01} \cap \mathfrak{z}_{\mathfrak{g}}(y)_{01}=\{0\}$.

Proof. A morphism of irreducible varieties is dominant if and only if its differential at some point is onto. As $\varphi$ is bilinear, an easy computation shows that $d \varphi_{(x, y)}(\xi, \eta)=$ $[x, \eta]+[\xi, y], \xi \in \mathfrak{g}_{10}, \eta \in \mathfrak{g}_{11}$. Hence $\operatorname{Im} d \varphi_{(x, y)}=\left[\mathfrak{g}_{11}, x\right]+\left[\mathfrak{g}_{10}, y\right]$, and taking 
the orthogonal complement with respect to the restriction of the Killing form to $\mathfrak{g}_{01}$ yields $\left(\operatorname{Im} d \varphi_{(x, y)}\right)^{\perp}=\mathfrak{z}_{\mathfrak{g}}(x)_{01} \cap \mathfrak{z}_{\mathfrak{g}}(y)_{01}$.

As we see below, certain CSS in $\mathfrak{g}_{1 \star}$ play an important role in describing irreducible components of $\mathfrak{E}$.

Definition 1. A big CSS $\mathfrak{c}_{1 \star} \subset \mathfrak{g}_{1 \star}$ is said to be homogeneous if it is $\sigma_{2}$-stable (or, equivalently, $\sigma_{3}$-stable). In other words, if one has $\mathfrak{c}_{1 \star}=\mathfrak{a}_{10} \oplus \mathfrak{a}_{11}$ with $\mathfrak{a}_{1 j} \subset \mathfrak{g}_{1 j}$.

Remark. A coincidence of CSS means that there is a homogeneous CSS of special form. For instance, if $\mathfrak{c}_{11}=\mathfrak{c}_{1 \star}$, then $\mathfrak{c}_{11}$ is a homogeneous CSS in $\mathfrak{g}_{1 \star}$, with trivial $\mathfrak{g}_{10}$-component.

Lemma 3.3. (1) Homogeneous CSS always exist.

(2) Moreover, if $x \in \mathfrak{g}_{10}$ and $y \in \mathfrak{g}_{11}$ are commuting semisimple elements, then there exists a homogeneous CSS in $\mathfrak{g}_{1 \star}$ containing both of them.

Proof. (1) Take a little CSS $\mathfrak{c}_{10}$ and consider the $\overrightarrow{\boldsymbol{\sigma}}$-stable reductive subalgebra $\mathfrak{z}_{\mathfrak{g}}\left(\mathfrak{c}_{10}\right)$. If $\tilde{\mathfrak{a}}_{11}$ is a little CSS in $\mathfrak{z}_{\mathfrak{g}}\left(\mathfrak{c}_{10}\right)_{11}$, then $\mathfrak{c}_{10} \oplus \tilde{\mathfrak{a}}_{11}$ is a homogeneous CSS in $\mathfrak{g}_{1 \star}$.

(2) Consider the $\overrightarrow{\boldsymbol{\sigma}}$-stable reductive subalgebra $\mathfrak{l}=\mathfrak{z}_{\mathfrak{g}}(x) \cap \mathfrak{z}_{\mathfrak{g}}(y)$. By the previous part, there exists a homogeneous CSS in $\mathfrak{l}_{1 \star}$, say $\tilde{\mathfrak{c}}_{1 \star}$. Since $x$ and $y$ are central in $\mathfrak{l}$, we have $x, y \in \tilde{\mathfrak{c}}_{1 \star}$. It is also clear that $\tilde{\mathfrak{c}}_{1 \star}$ is a CSS in $\mathfrak{g}_{1 \star}$.

If $\mathfrak{c}_{1 \star}=\mathfrak{a}_{10} \oplus \mathfrak{a}_{11}$ is a homogeneous CSS, then $\left[\mathfrak{a}_{01}, \mathfrak{a}_{11}\right]=0$ and hence $\overline{G_{00} \cdot \mathfrak{c}_{1 \star}} \subset$ $\mathfrak{E}$. However, a stronger result is true.

Theorem 3.4. (i) Let $\mathfrak{c}_{1 \star}$ be a homogeneous CSS in $\mathfrak{g}_{1 \star}$. Then $\overline{G_{00} \cdot \mathfrak{c}_{1 \star}} \subset \mathfrak{E}$ is an irreducible component of $\mathfrak{E}$.

(ii) If two homogeneous CSS in $\mathfrak{g}_{1 \star}$ are not $G_{00}$-conjugate, then the corresponding irreducible components are different.

Proof. (i) The centralizer of $\mathfrak{c}_{1 \star}$ is $\overrightarrow{\boldsymbol{\sigma}}$-stable. Hence $\mathfrak{z}_{\mathfrak{g}}\left(\mathfrak{c}_{1 \star}\right)=\bigoplus_{i, j=0,1} \mathfrak{a}_{i j}$, and here $\mathfrak{c}_{1 \star}=\mathfrak{a}_{10} \oplus \mathfrak{a}_{11}$. Recall that $\overline{G_{0 \star} \cdot \mathfrak{c}_{1 \star}}=\mathfrak{g}_{1 \star}$. Therefore, $\operatorname{dim} \mathfrak{c}_{1 \star}+\operatorname{dim} G_{0 \star}-\operatorname{dim} \mathfrak{a}_{00}-$ $\operatorname{dim} \mathfrak{a}_{01}=\operatorname{dim} \mathfrak{g}_{1 \star}$. It follows that

$$
\operatorname{dim} \overline{G_{00} \cdot \mathfrak{c}_{1 \star}}=\operatorname{dim} \mathfrak{c}_{1 \star}+\operatorname{dim} G_{00}-\operatorname{dim} \mathfrak{a}_{00}=\operatorname{dim} \mathfrak{g}_{1 \star}-\operatorname{dim} \mathfrak{g}_{01}+\operatorname{dim} \mathfrak{a}_{01} .
$$

On the other hand, let $x+y \in \mathfrak{c}_{1 \star}\left(x \in \mathfrak{g}_{10}, y \in \mathfrak{g}_{11}\right)$. The proof of Lemma 3.2 shows that $\operatorname{dim}\left(\operatorname{Im} d \varphi_{(x, y)}\right)=\operatorname{dim} \mathfrak{g}_{01}-\operatorname{dim}\left(\mathfrak{z}_{\mathfrak{g}}(x)_{01} \cap \mathfrak{z}_{\mathfrak{g}}(y)_{01}\right)$. Now, if $x+y \in \mathfrak{c}_{1 \star}$ is generic, then $\mathfrak{z}_{\mathfrak{g}}(x) \cap \mathfrak{z}_{\mathfrak{g}}(y)=\mathfrak{z}_{\mathfrak{g}}(x+y)=\mathfrak{z}_{\mathfrak{g}}\left(\mathfrak{c}_{1 \star}\right)$. Hence $\operatorname{dim}\left(\operatorname{Im} d \varphi_{(x, y)}\right)=$ $\operatorname{dim} \mathfrak{g}_{01}-\operatorname{dim} \mathfrak{a}_{01}$. This means that any irreducible component of $\mathfrak{E}$ containing $(x, y)$ has dimension at most

$$
\operatorname{dim} \mathfrak{g}_{1 \star}-\operatorname{dim}\left(\operatorname{Im} d \varphi_{(x, y)}\right)=\operatorname{dim} \mathfrak{g}_{1 \star}-\operatorname{dim} \mathfrak{g}_{01}+\operatorname{dim} \mathfrak{a}_{01} .
$$


Comparing with (3-2) shows that $\overline{G_{00} \cdot \mathfrak{c}_{1 \star}}$ is an irreducible component of E containing $(x, y)$, and $(x, y)$ is a smooth point of $\overline{G_{00} \cdot \mathfrak{c}_{1 \star}}$.

(ii) As we have just shown, if $x+y \in \mathfrak{c}_{1 \star}$ is generic, then it belongs to a unique irreducible component of $\mathfrak{E}$ (and to a unique CSS in $\mathfrak{g}_{1_{\star}}$ ).

Claim 3.5. The number of $G_{00}$-orbits of homogeneous CSS in $\mathfrak{g}_{1 \star}$ is finite.

First proof. Since the number of irreducible components is finite, this readily follows from Theorem 3.4. However, it can also be proved in a different way. As the second proof has its own merits, we provide it below.

Second proof. Recall that $G_{00} \subset G_{0 \star}$ are connected reductive groups and all big CSS in $\mathfrak{g}_{1 \star}$ form a single $G_{0 \star}$-orbit. Let $\mathfrak{c}_{1 \star}$ be a homogeneous CSS. Set

$$
N=\left\{g \in G_{0 \star} \mid g \cdot \mathfrak{c}_{1 \star}=\mathfrak{c}_{1 \star}\right\}, \quad \mathcal{M}=\left\{g \in G_{0 \star} \mid g \cdot \mathfrak{c}_{1 \star} \text { is homogeneous }\right\} .
$$

Note that $N$ is reductive, but not connected, since $N$ is mapped onto the (finite) little Weyl group associated with $\mathfrak{c}_{1 \star}$. If $g \in \mathcal{M}, s \in G_{00}$, and $z \in N$, then $s g z \in \mathcal{M}$. Therefore, $\mathcal{M}$ is a union of $\left(G_{00}, N\right)$-cosets, and our task is to prove that $G_{00} \backslash \mathcal{M} / N$ is finite.

If $g \in \mathcal{M}$, then $g \cdot \mathfrak{c}_{1 \star}=\sigma_{2}(g) \mathfrak{c}_{1 \star}$. Hence $g^{-1} \sigma_{2}(g) \in N$. Since $G_{00} \subset G^{\sigma_{2}}$, the map

$$
\psi_{\mathcal{M}}: G_{00} \backslash \mathcal{M} \rightarrow N, \quad G_{00} g \mapsto g^{-1} \sigma_{2}(g),
$$

is well defined. Note that $N$ is $\sigma_{2}$-stable and the range of $\psi_{\mathcal{M}}$ belongs to the closed subset

$$
\mathcal{Q}=\mathcal{Q}(N)=\left\{g \in N \mid \sigma_{2}(g)=g^{-1}\right\}
$$

The twisted $N$-action on $N$ is defined by $z \star x=z x \sigma_{2}(z)^{-1}$. Obviously, $\mathcal{Q}$ is stable under the twisted action of $N$. Moreover, $\psi_{\mathcal{M}}(g z)=z^{-1} \psi_{\mathcal{M}}(g) \sigma_{2}(z)$. Hence $\operatorname{Im}\left(\varphi_{\mathcal{M}}\right) \subset \mathcal{Q}$ is the union of twisted $N$-orbits, and each twisted $N$-orbit gives rise to a $G_{00}$-orbit of homogeneous CSS. It follows from [Richardson 1982, §9] that $\mathcal{Q}$ is a finite union of twisted $N$-orbits, which is sufficient for our purpose. (See also the remark below.)

Remark 3.6. Richardson's results on twisted orbits [Richardson 1982, §9], specifically Proposition 9.1, are stated for a connected reductive group $G$, whereas we apply them to the reductive nonconnected group $N$ (in place of $G$ ). But his argument can easily be adjusted to cover the case of nonconnected reductive groups. That is, one can give a version of Richardson's Proposition 9.1 for nonconnected groups $G$.

Definition 2. For a homogeneous CSS $\mathfrak{c}_{1 \star} \subset \mathfrak{g}_{1 \star}$, the irreducible component $\overline{G_{00} \cdot \mathfrak{c}_{1 \star}}$ of $\mathfrak{E}$ is said to be standard. 
Since all big CSS in $\mathfrak{g}_{1 \star}$ are $G_{0 \star}$-conjugate, their centralizers in $\mathfrak{g}_{0 \star}$ are essentially "the same". The centralizer in $\mathfrak{g}_{0 \star}$ of a homogeneous CSS splits, and these splittings can be quite different. That is, $\operatorname{dim} \mathfrak{z}_{\mathfrak{g}}\left(\mathfrak{c}_{1 \star}\right)_{01}$ can be different for different homogeneous CSS, and this leads to a new phenomenon that standard irreducible components of $\mathfrak{E}$ may have different dimensions, see (3-2). Moreover, there can also be some "nonstandard" irreducible components of $\mathfrak{E}$ that contain no semisimple elements at all.

By Theorem 3.4, a necessary condition for $\mathfrak{E}$ to be irreducible is that all homogeneous CSS in $\mathfrak{g}_{1 \star}$ are $G_{00}$-conjugate, that is, there is only one standard component. If $\mathfrak{c}_{1 \star}=\mathfrak{a}_{10} \oplus \mathfrak{a}_{11}$ is a homogeneous CSS with $\operatorname{dim} \mathfrak{a}_{1 i}=d_{i}$, then $\left(d_{0}, d_{1}\right)$ is called the dimension vector. Obviously, two homogeneous CSS with different dimension vectors are not $G_{00}$-conjugate.

Theorem 3.7. (1) If $\mathfrak{c}_{1 \star}=\mathfrak{a}_{10} \oplus \mathfrak{a}_{11}$ is a homogeneous CSS with dimension vector $\left(d_{0}, d_{1}\right)$, then $d_{0} \leqslant \operatorname{dim} \mathfrak{c}_{10}$ and $d_{1} \leqslant \operatorname{dim} \mathfrak{c}_{11}$; hence $\operatorname{dim} \mathfrak{c}_{1 \star} \leqslant \operatorname{dim} \mathfrak{c}_{10}+\operatorname{dim} \mathfrak{c}_{11}$.

(2) All homogeneous CSS in $\mathfrak{g}_{1 \star}$ are $G_{00}$-conjugate if and only if $\operatorname{dim} \mathfrak{c}_{1 \star}=\operatorname{dim} \mathfrak{c}_{10}+$ $\operatorname{dim} \mathfrak{c}_{11}$.

Proof. (1) Being a toral subalgebra of $\mathfrak{g}_{1 j}, \mathfrak{a}_{1 j}$ is contained in a little CSS in $\mathfrak{g}_{1 j}$.

(2) “If” part. Let $\mathfrak{c}_{1 \star}$ and $\tilde{\mathfrak{c}}_{1 \star}=\tilde{\mathfrak{a}}_{10} \oplus \tilde{\mathfrak{a}}_{11}$ be two homogeneous CSS. By part (1), $\operatorname{dim} \mathfrak{a}_{01}=\operatorname{dim} \tilde{\mathfrak{a}}_{01}=\operatorname{dim} \mathfrak{c}_{10}$. Therefore, both $\mathfrak{a}_{01}$ and $\tilde{\mathfrak{a}}_{01}$ are little CSS, they are both $G_{00}$-conjugate, and we may assume that $\mathfrak{a}_{01}=\tilde{\mathfrak{a}}_{01}$. Consider then the $\overrightarrow{\boldsymbol{\sigma}}$-stable reductive algebra $\mathfrak{z}_{\mathfrak{g}}\left(\mathfrak{a}_{10}\right)$. As $\mathfrak{a}_{10}$ is a central toral subalgebra, $\mathfrak{z}_{\mathfrak{g}}\left(\mathfrak{a}_{10}\right)=\mathfrak{a}_{10} \oplus \mathfrak{s}$, where $\mathfrak{s}$ is reductive and $\overrightarrow{\boldsymbol{\sigma}}$-stable. By construction, $\mathfrak{s}_{10}=\{0\}$ and $\mathfrak{a}_{11}, \tilde{\mathfrak{a}}_{11} \subset \mathfrak{s}_{11}$. Moreover, these are little CSS in $\mathfrak{s}_{11}$ (otherwise, $\mathfrak{c}_{1 \star}$ or $\tilde{\mathfrak{c}}_{1 \star}$ wouldn't be maximal). Therefore, $\mathfrak{a}_{01}$ and $\tilde{\mathfrak{a}}_{01}$ are $S_{00}$-conjugate, which implies that $\mathfrak{c}_{1 \star}$ and $\tilde{\mathfrak{c}}_{1 \star}$ and $G_{00^{-}}$ conjugate.

"Only if" part. Assuming that $\operatorname{dim} \mathfrak{c}_{1 \star}<\operatorname{dim} \mathfrak{c}_{10}+\operatorname{dim} \mathfrak{c}_{11}$, we construct two homogeneous CSS with different dimension vectors. First, let us take a little CSS $\mathfrak{c}_{10}$ and choose a little CSS in $\mathfrak{z}_{\mathfrak{g}}\left(\mathfrak{c}_{10}\right)_{11}$, say $\tilde{\mathfrak{a}}_{11}$. This yields a homogeneous CSS with dimension vector $\left(\operatorname{dim} \mathfrak{c}_{10}, \operatorname{dim} \mathfrak{c}_{1 \star}-\operatorname{dim} \mathfrak{c}_{10}\right)$. On the other hand, one can start with a little CSS $\mathfrak{c}_{11}$, etc., which yields a homogeneous CSS with dimension vector $\left(\operatorname{dim} \mathfrak{c}_{1 \star}-\operatorname{dim} \mathfrak{c}_{11}, \operatorname{dim} \mathfrak{c}_{11}\right)$.

Note that $\operatorname{dim} \mathfrak{c}_{i j}>0$ whenever $\mathfrak{g}_{i j} \neq\{0\}$. Therefore, a coincidence of CSS of the form $\mathfrak{c}_{11}=\mathfrak{c}_{1 \star}$ or $\mathfrak{c}_{10}=\mathfrak{c}_{1 \star}$ certainly excludes the possibility of having a unique standard component of $\mathfrak{E}$. For our test commutator (3-1), one may envisage several examples of good behavior (not necessarily all together):

(1) All irreducible components of $\mathfrak{E}$ are standard (possibly of different dimension).

(2) $\varphi$ is surjective and equidimensional, and hence flat.

(3) $\mathfrak{E}$ has a unique standard component, but there may be other components too. 
Property (3) always holds in the setting of Example 3.1, with any $\sigma$; and for $\sigma$ of maximal rank, one gets a rare situation, where all three properties are satisfied. All quaternionic decompositions of simple Lie algebras can be written out explicitly, and then the presence of (3) amounts to a routine verification of the equality in Theorem 3.7(2).

Proposition 3.8. Let $\left\{\sigma_{1}, \sigma_{2}\right\}$ be a dyad. Then $\operatorname{dim} \mathfrak{g}_{10}=\operatorname{dim} \mathfrak{g}_{01}$ and $\varphi: \mathfrak{g}_{10} \times \mathfrak{g}_{11} \rightarrow$ $\mathfrak{g}_{01}$ is onto. (Therefore, $\operatorname{dim} \varphi^{-1}(\xi) \geqslant \operatorname{dim} \mathfrak{g}_{11}$ for all $\xi \in \mathfrak{g}_{01}$.) Moreover, $\{0\} \times \mathfrak{g}_{11}$ is a standard irreducible component of $\mathfrak{E}$ of minimal dimension.

Proof. Since $\operatorname{dim} \mathfrak{g}^{\sigma_{1}}=\operatorname{dim} \mathfrak{g}^{\sigma_{2}}$, we have $\operatorname{dim} \mathfrak{g}_{10}=\operatorname{dim} \mathfrak{g}_{01}$. By Theorem 2.2(2), any little CSS $\mathfrak{c}_{11} \subset \mathfrak{g}_{11}$ is also a big CSS in $\mathfrak{g}_{1 \star}$. Therefore, $\mathfrak{c}_{11}$ is a homogeneous CSS and $\overline{G_{00} \cdot \mathfrak{c}_{11}}=\mathfrak{g}_{11}$ is an irreducible component of $\mathfrak{E}$. Furthermore, if $x \in \mathfrak{c}_{11}$ is generic, then $\mathfrak{z}_{\mathfrak{g}}(x) \cap \mathfrak{g}_{1 \star}=\mathfrak{c}_{11}$, that is, $\mathfrak{z}_{\mathfrak{g}}(x) \cap \mathfrak{g}_{10}=\{0\}$. Therefore, $\operatorname{dim}\left[\mathfrak{g}_{10}, x\right]=$ $\operatorname{dim} \mathfrak{g}_{10}$, that is, $\left[\mathfrak{g}_{10}, x\right]=\mathfrak{g}_{01}$.

\section{Dyads of maximal rank and commuting varieties}

Let $\left\{\sigma_{1}, \sigma_{2}\right\}$ be a dyad of maximal rank, that is, both $\sigma_{1}$ and $\sigma_{2}$ are of maximal rank. Recall that this implies that $\sigma_{3}=\sigma_{1} \sigma_{2}$ is inner, $\operatorname{dim} \mathfrak{g}_{01}=\operatorname{dim} \mathfrak{g}_{10}$, and, by Proposition 2.1, $\mathfrak{g}^{\sigma_{3}}=\mathfrak{g}_{00} \oplus \mathfrak{g}_{11}$ is a $\mathbb{Z}_{2}$-grading of maximal rank. In particular, $\mathfrak{g}_{11}$ contains a CSA of $\mathfrak{g}$ and any CSS in $\mathfrak{g}_{1 \star}$ or $\mathfrak{g}_{\star}$ is a CSA. The main result of this section is the following.

Theorem 4.1. Let $\left\{\sigma_{1}, \sigma_{2}\right\}$ be a dyad of maximal rank. Then

(i) the commutator mapping $\varphi: \mathfrak{g}_{10} \times \mathfrak{g}_{11} \rightarrow \mathfrak{g}_{01}$ is surjective and equidimensional,

(ii) each irreducible component of $\mathfrak{E}=\varphi^{-1}(0)$ is standard, that is, is the closure of the $G_{00}$-saturation of a homogeneous CSS in $\mathfrak{g}_{1 \star}$, and

(iii) the ideal of $\mathfrak{E}$ is generated by quadrics $\varphi^{\#}\left(\mathfrak{g}_{01}^{*}\right)$, where $\varphi^{\#}: \mathbb{k}\left[\mathfrak{g}_{01}\right] \rightarrow \mathbb{k}\left[\mathfrak{g}_{10}\right] \otimes$ $\mathbb{k}\left[\mathfrak{g}_{11}\right]$ is the comorphism (that is, the scheme $\varphi^{-1}(0)$ is a reduced complete intersection).

Proof. If $\mathfrak{q}$ is a $\overrightarrow{\boldsymbol{\sigma}}$-stable reductive subalgebra of $\mathfrak{g}$, then $\mathfrak{E}_{\mathfrak{q}}$ stands for the zero fiber of the commutator $\mathfrak{q}_{10} \times \mathfrak{q}_{11} \rightarrow \mathfrak{q}_{01}$. Clearly, $\mathfrak{E}_{q} \subset \mathfrak{E}=\mathfrak{E}_{\mathfrak{g}}$. Since $\sigma_{1}$ and $\sigma_{2}$ are of maximal rank, the center of $\mathfrak{g}, \mathfrak{z}(\mathfrak{g})$, is contained in $\mathfrak{g}_{11}$. Consequently, $\mathfrak{E}_{\mathfrak{g}} \simeq \mathfrak{E}_{[\mathfrak{g}, \mathfrak{g}]} \times \mathfrak{z}(\mathfrak{g})$ and without loss of generality, we may assume that $\mathfrak{g}$ is semisimple.

By Proposition 3.8, $\varphi$ is onto and $\operatorname{dim} \mathfrak{E} \geqslant \operatorname{dim} \mathfrak{g}_{11}$. In this situation, $\varphi$ is equidimensional if and only if $\operatorname{dim} \mathfrak{E}=\operatorname{dim} \mathfrak{g}_{11}$. If $\mathfrak{c}_{1 \star}$ is a homogeneous CSS, then it is necessarily a CSA of $\mathfrak{g}$. That is, $\mathfrak{z}_{\mathfrak{g}}\left(\mathfrak{c}_{1 \star}\right)_{01}=0$ for all homogeneous CSS and $\operatorname{dim} \overline{G_{00} \cdot \mathfrak{c}_{1 \star}}=\operatorname{dim} \mathfrak{g}_{11}$. Hence all the standard components of $\mathfrak{E}$ have the same (expected) dimension, and for (i) and (ii) it suffices to prove that there are no other irreducible components.

To this end, we argue by induction on $r k \mathfrak{g}=\operatorname{dim} \mathfrak{c}_{11}$. 
- If $\operatorname{dim} \mathfrak{c}_{11}=1$, then $\mathfrak{g}=\mathfrak{s l}_{2}$ and the assertion is true.

- Suppose that $r k \mathfrak{g}>1$ and the assertion holds for all dyads of maximal rank for semisimple algebras of rank smaller than $r k \mathfrak{g}$.

(1) Take $(x, y) \in \mathfrak{E}$ and $y \in \mathfrak{g}_{11}$, and let $y=y_{s}+y_{n}$ be the Jordan decomposition. Then $\left[x, y_{s}\right]=0$. If $y_{s} \neq 0$, then $y_{n} \in \mathfrak{s}:=\left[\mathfrak{z}_{\mathfrak{g}}\left(y_{s}\right), \mathfrak{z}_{\mathfrak{g}}\left(y_{s}\right)\right]$ and $\mathrm{rk} \mathfrak{s}<\mathrm{rk} \mathfrak{g}$. By Lemma $1.2,\left.\sigma_{i}\right|_{\mathfrak{s}}, i=1,2$, are again involutions of maximal rank. Let $\mathfrak{z}$ denote the center of $\mathfrak{z}_{\mathfrak{g}}\left(y_{s}\right)$, so that $\mathfrak{z}_{\mathfrak{g}}\left(y_{s}\right)=\mathfrak{z} \oplus \mathfrak{s}$ and $y_{s} \in \mathfrak{z}$. Since both $\sigma_{1}$ and $\sigma_{2}$ are of maximal rank, $\mathfrak{z} \subset \mathfrak{g}_{11}$ and hence $x \in \mathfrak{s}$. By the induction assumption, $\left(x, y_{n}\right) \in \mathfrak{s}_{10} \oplus \mathfrak{s}_{11}$ lies in a standard irreducible component of $\mathfrak{E}_{\mathfrak{s}}$. Obviously, adding a central summand does not affect this property, hence $(x, y)$ lies in a standard component of $\mathfrak{E}_{\mathfrak{g} \mathfrak{g}\left(y_{s}\right)}$. As $r k \mathfrak{z} \mathfrak{g}\left(y_{s}\right)=\mathrm{rk} \mathfrak{g}$, this also means that $(x, y)$ lies in a standard component of $\mathfrak{E}$.

(2) Hence it suffices to consider the case in which $y=y_{n}$. Write $\mathcal{N}_{11}$ for the closed set of all nilpotent elements in $\mathfrak{g}_{11}$. Let $\mathcal{K}$ be an irreducible component of $\mathfrak{E}$, hence $\operatorname{dim} \mathcal{K} \geqslant \operatorname{dim} \mathfrak{g}_{11}$. Then $\mathcal{K}_{1}:=\mathcal{K} \cap\left(\mathfrak{g}_{10} \times \mathcal{N}_{11}\right)$ is a closed subvariety of $\mathcal{K}$. If $\mathcal{K}_{1} \neq \mathcal{K}$, then, by part (1), all the points in $\mathcal{K} \backslash \mathcal{K}_{1}$ belong to standard irreducible components. Consequently, $\mathcal{K}$ must be one of the standard components.

(3) The next possibility is that $\mathcal{K}=\mathcal{K}_{1}$. Let $p: \mathfrak{g}_{10} \times \mathfrak{g}_{11} \rightarrow \mathfrak{g}_{11}$ be the projection. Then $p(\mathcal{K}) \subset \mathcal{N}_{11}$, and therefore $\overline{p(\mathcal{K})}=\overline{G_{00} \cdot y}$ is the closure of a nilpotent $G_{00}$-orbit.

If $y=0$, then $\mathcal{K}=\mathfrak{g}_{10} \times\{0\}$. Let $\mathfrak{c}_{10}$ be a little CSS. The fact that $\overline{G_{00} \cdot\left(\mathfrak{c}_{10} \times\{0\}\right)}=$ $\mathfrak{g}_{10} \times\{0\}$ is an irreducible component of $\mathfrak{E}$ implies that $\mathfrak{z}_{\mathfrak{g}}\left(\mathfrak{c}_{10}\right)_{11}=\{0\}$, whence $\mathfrak{c}_{10}$ is also a CSS in $\mathfrak{g}_{1 \star}$. That is, $\mathfrak{c}_{10}$ is a CSA of $\mathfrak{g}$. (Incidentally, this means that the (-1)-eigenspace of $\sigma_{3}$ contains a CSA, that is, $\left\{\sigma_{1}, \sigma_{2}, \sigma_{3}\right\}$ is actually a triad.) Anyway, we see that if $y=0$, then such $\mathcal{K}$ appears to be a standard component.

(4) Finally, we prove that the case in which $\mathcal{K}=\mathcal{K}_{1}$ and $y \neq 0$ is impossible. Assuming the contrary, we would have

$$
\begin{aligned}
& \operatorname{dim} \mathfrak{g}_{11} \leqslant \operatorname{dim} \mathcal{K} \leqslant \operatorname{dim} G_{00} \cdot y+\operatorname{dim} p^{-1}(y) \\
& \quad=\operatorname{dim} \mathfrak{g}_{00}-\operatorname{dim}_{\mathfrak{z} \mathfrak{g}}(y)_{00}+\operatorname{dim} \mathfrak{z}_{\mathfrak{g}}(y)_{10}=\operatorname{dim} \mathfrak{g}_{11}-\operatorname{dim} \mathfrak{z}_{\mathfrak{g}}(y)_{11}+\operatorname{dim} \mathfrak{z}_{\mathfrak{g}}(y)_{10} .
\end{aligned}
$$

The last equality uses Lemma 1.1 . Hence, the existence of such a component $\mathcal{K}$ would imply that $\operatorname{dim}_{\mathfrak{z} \mathfrak{g}}(y)_{11} \leqslant \operatorname{dim} \mathfrak{z}_{\mathfrak{g}}(y)_{10}$ for some nonzero $y \in \mathcal{N}_{11} \subset \mathfrak{g}_{11}$. One can rewrite the last condition so that it will only depend on the (inner) involution $\sigma_{3}$. Since $\left\{\sigma_{1}, \sigma_{2}\right\}$ is a dyad, we have $\operatorname{dim} \mathfrak{z}_{\mathfrak{g}}(y)_{10}=\operatorname{dim} \mathfrak{z}_{\mathfrak{g}}(y)_{01}$; and since $\sigma_{3}$ is inner and $\mathfrak{g}^{\sigma_{3}}=\mathfrak{g}_{00} \oplus \mathfrak{g}_{11}$ is a $\mathbb{Z}_{2}$-grading of maximal rank, we have $\operatorname{dim} \mathfrak{z}_{\mathfrak{g}}(y)_{11}=$ $\operatorname{dim} \mathfrak{z}_{\mathfrak{g}}(y)_{00}+\mathrm{rk} \mathfrak{g}^{\sigma_{\mathfrak{3}}}=\operatorname{dim} \mathfrak{z}_{\mathfrak{g}}(y)_{00}+\mathrm{rk} \mathfrak{g}$, see (1-2). Then

$\operatorname{dim} \mathfrak{z}_{\mathfrak{g}}(y)_{11}+\operatorname{dim} \mathfrak{z}_{\mathfrak{g}}(y)_{00}+\mathrm{rk} \mathfrak{g}=2 \operatorname{dim} \mathfrak{z}_{\mathfrak{g}}(y)_{11} \leqslant 2 \operatorname{dim} \mathfrak{z}_{\mathfrak{g}}(y)_{10}$

$$
=\operatorname{dim}_{\mathfrak{z} \mathfrak{g}}(y)_{10}+\operatorname{dim} \mathfrak{z}_{\mathfrak{g}}(y)_{01} \text {. }
$$


In other words, if the assumption were true, we would have

$$
\operatorname{dim}\left(\mathfrak{z}_{\mathfrak{g}}(y) \cap \mathfrak{g}^{\sigma_{3}}\right)+\mathrm{rk} \mathfrak{g} \leqslant \operatorname{dim}\left(\mathfrak{z}_{\mathfrak{g}}(y) \cap \mathfrak{g}_{1}^{\left(\sigma_{3}\right)}\right)
$$

for some nonzero nilpotent $y \in \mathfrak{g}_{11}$. (Note that since $\mathfrak{g}^{\sigma_{3}}=\mathfrak{g}_{00} \oplus \mathfrak{g}_{11}$ is a $\mathbb{Z}_{2}$-grading of maximal rank, $\mathfrak{g}_{11}$ meets all nilpotent orbits in $\mathfrak{g}^{\sigma_{3}}$ [Antonyan 1982]. Therefore, a priori, $y$ can be any nonzero nilpotent element of $\mathfrak{g}^{\sigma_{3}}$.) However, Theorem 4.4 shows that (4-1) is never satisfied if $y \neq 0$. This completes the proof of parts (i) and (ii).

For (iii), it suffices to prove that each irreducible component of $\mathfrak{E}$ contains a point $(x, y)$ such that $d \varphi_{(x, y)}$ is onto, that is, $\operatorname{Im} d \varphi_{(x, y)}=\mathfrak{g}_{01}$, see [Richardson 1981, Lemma 2.3]. Since each irreducible component of $\mathfrak{E}$ is the closure of the $G_{00}$-saturation of a homogeneous CSA, it contains a point $(x, y)$ such that $\mathfrak{z}_{\mathfrak{g}}(x)_{01} \cap$ $\mathfrak{z}_{\mathfrak{g}}(y)_{01}=\{0\}$ and then $d \varphi_{(x, y)}$ is onto, as shown in the proof of Lemma 3.2.

Remark 4.2. (1) For any inner $\sigma \in \operatorname{lnv}(\mathfrak{g})$, there exist commuting involutions of maximal rank $\sigma_{1}$ and $\sigma_{2}$ such that $\sigma=\sigma_{1} \sigma_{2}$, see Proposition 2.1. Therefore, there are sufficiently many quaternionic decompositions, where Theorem 4.1 applies.

(2) For an arbitrary dyad $\left\{\sigma_{1}, \sigma_{2}\right\}$, it can happen that all irreducible components of $\mathfrak{E}$ are standard, but they have different dimensions. That is, $\varphi: \mathfrak{g}_{10} \times \mathfrak{g}_{11} \rightarrow \mathfrak{g}_{01}$ is not equidimensional, but still any pair of commuting elements in $\mathfrak{g}_{10} \times \mathfrak{g}_{11}$ can be approximated by a pair of commuting semisimple elements.

Example 4.3. Let $\sigma_{1}$ be an involution of $\mathfrak{g}=\mathfrak{s o}_{n}$ such that $\mathfrak{g}^{\sigma_{1}}=\mathfrak{s o}_{n-1}$. This can be included in a dyad $\left\{\sigma_{1}, \sigma_{2}\right\}$ such that $\mathfrak{g}^{\sigma_{3}}=\mathfrak{s o}_{n-2} \times \mathfrak{s o}_{2}$. The quaternionic decomposition is

$$
\mathfrak{g}=\frac{\mathfrak{s o}_{n-2}}{\mathrm{R}\left(\varpi_{1}\right)} \underset{\sigma_{2}}{\mathrm{R}\left(\varpi_{1}\right)} \mathrm{R}(0) \sigma_{1},
$$

where the trivial $\mathfrak{s o}_{n-2}$-module $\mathrm{R}(0)$ is just the central torus $\mathfrak{s o}_{2}$ in $\mathfrak{g}^{\sigma_{3}}$. Here $\operatorname{dim} \mathfrak{c}_{10}=\operatorname{dim} \mathfrak{c}_{11}=1$ and the zero fiber of multiplication $\mathfrak{g}_{10} \times \mathfrak{g}_{11} \rightarrow \mathfrak{g}_{01}$ consists of two irreducible components, $\mathfrak{g}_{10} \times\{0\} \simeq \mathbb{k}^{n-2}$ and $\{0\} \times \mathfrak{g}_{11} \simeq \mathbb{k}$. Both components are standard.

The following auxiliary result does not refer to quaternionic decompositions; it concerns the case of a sole involution.

Theorem 4.4. Let $\sigma$ be an arbitrary involution of $\mathfrak{g}$ and $\mathfrak{g}=\mathfrak{g}_{0} \oplus \mathfrak{g}_{1}$ the corresponding $\mathbb{Z}_{2}$-grading. For any nonzero $x \in \mathfrak{g}_{0}$, we have

$$
\operatorname{dim} \mathfrak{g}_{0}^{x}+\mathrm{rk} \mathfrak{g}-\operatorname{dim} \mathfrak{g}_{1}^{x}>0
$$


More precisely, one always has $\operatorname{dim} \mathfrak{g}_{0}^{x}+\mathrm{rk} \mathfrak{g}-\operatorname{dim} \mathfrak{g}_{1}^{x} \geqslant 0$ and the equality only occurs if $x=0$ and $\sigma$ is of maximal rank.

Remark 4.5. For application to Theorem 4.1, we only need the case when $x$ is nilpotent and $\sigma$ is inner. But, surprisingly, the assertion appears to be absolutely general. Unfortunately, our proof is not conceptual, after all. Having successfully reduced the problem to noneven nilpotent elements of $\mathfrak{g}_{0}$, we then resort to caseby-case considerations. Certainly, there must be a better proof!

Proof. Note that $\operatorname{dim} G \cdot x$ is even and, therefore, the left-hand side in (4-2) is always even; hence the more accurate assertion is that $\operatorname{dim} \mathfrak{g}_{0}^{x}+\mathrm{rk} \mathfrak{g}-\operatorname{dim} \mathfrak{g}_{1}^{x} \geqslant 2$ for all nonzero $x \in \mathfrak{g}_{0}$.

(1) If $x=0$, then we have $\operatorname{dim} \mathfrak{g}_{0}+\mathrm{rk} \mathfrak{g}-\operatorname{dim} \mathfrak{g}_{1} \geqslant 0$, and the equality holds if and only if $\sigma$ is of maximal rank.

(2) If $x$ is nonzero semisimple, then $\mathfrak{g}^{x}$ is a $\sigma$-stable reductive subalgebra and $x$ is a central element of $\mathfrak{g}^{x}$ that belongs to $\mathfrak{g}_{0}^{x}$. Write $\mathfrak{g}^{x}=\mathfrak{z} \oplus \mathfrak{s}$, where $\mathfrak{s}=\left[\mathfrak{g}^{x}, \mathfrak{g}^{x}\right]$ and $\mathfrak{z}$ is the center. Then $\operatorname{dim} \mathfrak{z}_{0}>0$ and

$$
\operatorname{dim} \mathfrak{g}_{0}^{x}+\mathrm{rk} \mathfrak{g}-\operatorname{dim} \mathfrak{g}_{1}^{x}=\left(\operatorname{dim} \mathfrak{s}_{0}+\mathrm{rk} \mathfrak{s}-\operatorname{dim} \mathfrak{s}_{1}\right)+2 \operatorname{dim} \mathfrak{z}_{0} \geqslant 2 .
$$

(3) If $x$ is nonnilpotent, then using the Jordan decomposition $x=x_{s}+x_{n}$, we reduce the problem to the same property for the nilpotent element $x_{n}$ in the $\sigma$-stable reductive subalgebra $\mathfrak{z}_{\mathfrak{g}}\left(x_{s}\right)$.

(4) From now on, we assume that $x=e \in \mathfrak{g}_{0}$ is nonzero and nilpotent. Choose an $\mathfrak{s l}_{2}$-triple $\{e, h, f\} \subset \mathfrak{g}_{0}$. Suppose that $e$ is even in $\mathfrak{g}$, that is, the eigenvalues of ad $h$ in $\mathfrak{g}$ are even. Then $\operatorname{dim} \mathfrak{g}^{h}=\operatorname{dim} \mathfrak{g}^{e}$ and $\operatorname{dim} \mathfrak{g}_{0}^{h}=\operatorname{dim} \mathfrak{g}_{0}^{e}$. Thus, the assertion is reduced to the same assertion for $h \in \mathfrak{g}_{0}$ and we are again in the setting of part (2).

(5) Suppose that $e$ is even in $\mathfrak{g}_{0}$, but not in $\mathfrak{g}$. That is, the eigenvalues of ad $h$ in $\mathfrak{g}_{0}$ are even, but ad $h$ has also some odd eigenvalues in $\mathfrak{g}_{1}$. Decomposing $\mathfrak{g}$ into the sum of $\sigma$-stable ideals, we may assume that either $\mathfrak{g}$ is simple or $\mathfrak{g}=\mathfrak{s} \oplus \mathfrak{s}$, where $\mathfrak{s}$ is simple and $\sigma$ is the permutation involution. In the second case, if $e$ is even in $\mathfrak{g}_{0}=\Delta(\mathfrak{s})$, then $e$ is also even in $\mathfrak{g}$. Therefore, without loss of generality, we may assume that $\mathfrak{g}$ is simple.

Let us decompose $\mathfrak{g}_{1}$ according to the parity of ad $h$-eigenvalues: $\mathfrak{g}_{1}=\mathfrak{g}_{1}^{\text {odd }} \oplus \mathfrak{g}_{1}^{\text {even }}$. By assumption, $\mathfrak{g}_{1}^{\text {odd }} \neq 0$. Then $\tilde{\mathfrak{g}}:=\left[\mathfrak{g}_{1}^{\text {odd }}, \mathfrak{g}_{1}^{\text {odd }}\right] \oplus \mathfrak{g}_{1}^{\text {odd }}$ is an ideal of $\mathfrak{g}$ that does not meet $\mathfrak{g}_{1}^{\text {even }}$. Therefore, $\tilde{\mathfrak{g}}=\mathfrak{g}$ and $\mathfrak{g}_{1}^{\text {even }}=0$. Hence $\mathfrak{g}_{0}^{e}=\left(\mathfrak{g}^{e}\right)^{\text {even }}$ and $\mathfrak{g}_{1}^{e}=\left(\mathfrak{g}^{e}\right)^{\text {odd }}$. Consider the $\mathbb{Z}$-grading of $\mathfrak{g}$ determined by the eigenvalues of $h, \mathfrak{g}=\bigoplus_{i \in \mathbb{Z}} \mathfrak{g}(i)$. The $\mathfrak{s l}_{2}$-theory shows that $\operatorname{dim}\left(\mathfrak{g}^{e}\right)^{\text {even }}=\operatorname{dim} \mathfrak{g}(0)$ and $\operatorname{dim}\left(\mathfrak{g}^{e}\right)^{\text {odd }}=\operatorname{dim} \mathfrak{g}(1)$. Hence $\operatorname{dim} \mathfrak{g}_{0}^{e}=\operatorname{dim} \mathfrak{g}(0)$ and $\operatorname{dim} \mathfrak{g}_{1}^{e}=\operatorname{dim} \mathfrak{g}(1)$. Finally, it follows from Vinberg's lemma [Vinberg 1976, §2.3] that the group $G(0)$ has finitely many orbits in $\mathfrak{g}(1)$, whence 
$\operatorname{dim} \mathfrak{g}(1) \leqslant \operatorname{dim} \mathfrak{g}(0)$. Thus, in this case the stronger inequality $\operatorname{dim} \mathfrak{g}_{0}^{e} \geqslant \operatorname{dim} \mathfrak{g}_{1}^{e}$ holds.

(6) Thus, it remains to handle the case in which a nilpotent element $e \in \mathfrak{g}_{0}$ is not even. Here we do not know an a priori argument and resort to the case-by-case considerations.

(7) If $\mathfrak{g}$ is a classical Lie algebra, then the nilpotent orbits in $\mathfrak{g}$ and $\mathfrak{g}_{0}$ are parameterised by partitions, and we use the explicit formulae for $\operatorname{dim} \mathfrak{g}^{e}$ and $\operatorname{dim} \mathfrak{g}_{0}^{e}$ in terms of partitions. Some of these calculations are presented in the Appendix.

(8) If $\mathfrak{g}$ is an exceptional simple Lie algebra, then, for any noneven nilpotent element $e \in \mathfrak{g}_{0}$, we determine the corresponding nilpotent orbit in $\mathfrak{g}$ and then compare the dimensions of $\mathfrak{g}_{0}^{e}$ and $\operatorname{dim} \mathfrak{g}^{e}$. While rather boring, the verification is, however, not very difficult.

For $\sigma$ inner, we use the seminal work [Dynkin 1952, Tables 16-20], in which Dynkin computed, for all simple three-dimensional subalgebras in exceptional Lie algebras, the "minimal including regular semisimple subalgebras" and the corresponding weighted Dynkin diagrams. See also comments on this article in [Dynkin 2000, pp. 309-312], where a few errors occurring in [Dynkin 1952] are corrected.

To convey the idea, consider some examples related to an (inner) involution of $\mathfrak{g}=\mathbf{E}_{8}$ with $\mathfrak{g}_{0}=\mathbf{D}_{8}=\mathfrak{s o}_{16}$. There are 33 noneven nilpotent orbits in $\mathfrak{g}_{0}$. (Recall that $e \in \mathfrak{s o}_{16}$ is noneven if and only if the partition of $e$ contains both odd and even parts.)

(a) Let $e \in \mathfrak{s o}_{16}$ be a nilpotent element corresponding to the partition $(11,2,2,1)$. Using [Hesselink 1976, Corollary 3.8(a)] or [Kraft and Procesi 1982, Proposition 2.4], we obtain $\operatorname{dim} \mathfrak{g}_{0}^{e}=16$. This partition also shows that a minimal including regular semisimple subalgebra of $\mathbf{D}_{8}$ containing $e$ is of type $\mathbf{D}_{6}+\mathbf{A}_{1}$. (Here $(11,1)$ is the partition of the regular nilpotent element of $\mathbf{D}_{6}$ and any pair of equal parts $(n, n)$ gives rise to the simple summand $\mathbf{A}_{n-1}$.) Then using [Dynkin 1952, Table 20], we detect the simple three-dimensional subalgebra in $\mathbf{E}_{8}$ with minimal including regular semisimple subalgebra of type $\mathbf{D}_{6}+\mathbf{A}_{1}$. The corresponding nilpotent orbit has the modern label $\mathbf{E}_{7}\left(a_{3}\right)$ and here $\operatorname{dim} \mathfrak{g}^{e}=28$. Hence $\operatorname{dim} \mathfrak{g}_{1}^{e}=12$ and (4-2) holds.

(b) Let $e \in \mathfrak{s o}_{16}$ correspond to the partition $(7,5,2,2)$. By [Hesselink 1976, Corollary 3.8(a)], $\operatorname{dim} \mathfrak{g}_{0}^{e}=22$. Here a minimal including regular semisimple subalgebra is of type $\mathbf{D}_{6}\left(a_{2}\right)+\mathbf{A}_{1}$, because the partition $(7,5)$ determines the distinguished nilpotent orbit in $\mathbf{D}_{6}$, which is denoted by $\mathbf{D}_{6}\left(a_{2}\right)$. Using [Dynkin 1952, Table 20], we detect the corresponding nilpotent orbit in $\mathfrak{g}$. This orbit is denoted nowadays by $\mathbf{E}_{7}\left(a_{5}\right)$ and here $\operatorname{dim} \mathfrak{g}^{e}=42$. 
(c) Let $e \in \mathfrak{s o}_{16}$ correspond to the partition $(7,4,4,1)$. By [Hesselink 1976, Corollary 3.8(a)], $\operatorname{dim} \mathfrak{g}_{0}^{e}=22$. Here a minimal including regular semisimple subalgebra is of type $\mathbf{D}_{4}+\mathbf{A}_{3}$. Using [Dynkin 1952, Table 20], we detect the corresponding nilpotent orbit in $\mathfrak{g}$. This orbit is denoted nowadays by $\mathbf{D}_{6}\left(a_{2}\right)$ and here $\operatorname{dim} \mathfrak{g}^{e}=44$. If $\sigma$ is outer, then $\mathfrak{g}$ is of type $\mathbf{E}_{6}$. In the respective two cases, we use the information on $e \in \mathfrak{g}_{0}$ for decomposing $\mathfrak{g}_{1}$ as a $\langle e, h, f\rangle$-module, which allows us to compute $\operatorname{dim} \mathfrak{g}_{1}^{e}$.

A case-free proof of Theorem 4.4 might be derived from the following conjectural invariant-theoretic property of centralizers. Recall that $\mathfrak{g}=\mathfrak{g}_{0} \oplus \mathfrak{g}_{1}$ and $e \in \mathfrak{g}_{0}$. Let $G_{0}^{e}$ be the connected subgroup of $G_{0}$ with Lie algebra $\mathfrak{g}_{0}^{e}$. Then $G_{0}^{e}$ acts on $\left(\mathfrak{g}_{1}^{e}\right)^{*}$ and we write $\mathbb{k}\left(\left(\mathfrak{g}_{1}^{e}\right)^{*}\right)^{G_{0}^{e}}$ for the field of $G_{0}^{e}$-invariant rational functions on $\left(\mathfrak{g}_{1}^{e}\right)^{*}$.

Conjecture 4.6. For any $e \in \mathfrak{g}_{0} \cap \mathcal{N}$, we have $\operatorname{trdeg} \mathbb{k}\left(\left(\mathfrak{g}_{1}^{e}\right)^{*}\right)^{G_{0}^{e}} \leqslant \mathrm{rk} \mathfrak{g}$.

By Rosenlicht's theorem [Brion 2000, Chapter I.6],

$$
\operatorname{trdeg} \mathbb{k}\left(\left(\mathfrak{g}_{1}^{e}\right)^{*}\right)^{G_{0}^{e}}=\operatorname{dim} \mathfrak{g}_{1}^{e}-\max _{\xi \in\left(\mathfrak{g}_{1}^{e}\right)^{*}} \operatorname{dim} G_{0}^{e} \cdot \xi .
$$

If $e \neq 0$, then the one-dimensional unipotent group $\exp (t e), t \in \mathbb{k}$, acts trivially on $\mathfrak{g}_{1}^{e}$ and hence $\max _{\xi \in\left(\mathfrak{g}_{1}^{e}\right)^{*}} \operatorname{dim} G_{0}^{e} \cdot \xi \leqslant \operatorname{dim} \mathfrak{g}_{0}^{e}-1$. Therefore, if the conjecture were true, we would obtain $\operatorname{dim} \mathfrak{g}_{1}^{e}-\operatorname{dim} \mathfrak{g}_{0}^{e}+1 \leqslant \mathrm{rk} \mathfrak{g}$, as required. Perhaps, this can be related to the Elashvili conjecture, which asserts that $\operatorname{trdeg} \mathbb{k}\left(\left(\mathfrak{g}^{e}\right)^{*}\right)^{G^{e}}=\mathrm{rk} \mathfrak{g}$ for all $e \in \mathcal{N}$.

Remark 4.7. Inequality (4-2) can be written as $\operatorname{dim} \mathfrak{g}_{0}^{x}>\operatorname{dim} \mathcal{B}_{x}$, where $\mathcal{B}_{x}$ is the variety of Borel subalgebras of $\mathfrak{g}$ containing $x$ (the Springer fiber of $x$ ). (Recall that $\left.\operatorname{dim} \mathcal{B}_{x}=\left(\operatorname{dim} \mathfrak{g}^{x}-\mathrm{rk} \mathfrak{g}\right) / 2.\right)$

\section{Commuting varieties and restricted root systems}

Here we assume that $\left\{\sigma_{1}, \sigma_{2}\right\}$ is a dyad. As above, we consider the commutator map $\varphi: \mathfrak{g}_{10} \times \mathfrak{g}_{11} \rightarrow \mathfrak{g}_{01}$ and the $\overrightarrow{\boldsymbol{\sigma}}$-commuting variety $\mathfrak{E}=\varphi^{-1}(0)$. Then $\operatorname{dim} \mathfrak{E} \geqslant \operatorname{dim} \mathfrak{g}_{11}$ and $\mathfrak{E}$ has a standard irreducible component of expected dimension dim $\mathfrak{g}_{11}$; namely, $\{0\} \times \mathfrak{g}_{11}$, see Proposition 3.8.

In this section, we describe a method for detecting subvarieties of $\mathfrak{E}$ of large dimension. This method is based on comparing restricted root systems for little and big symmetric spaces related to the quaternionic decomposition in question.

Take a little CSS $\mathfrak{c}_{11} \subset \mathfrak{g}_{11}$. Then, by Theorem 2.2(2), $\mathfrak{c}_{11}$ is also a CSS in $\mathfrak{g}_{1 \star}$ and $\mathfrak{g}_{\star 1}$, which is equivalent to that $\mathfrak{z}_{\mathfrak{g}}\left(\mathfrak{c}_{11}\right)_{10}=\mathfrak{z}_{\mathfrak{g}}\left(\mathfrak{c}_{11}\right)_{01}=\{0\}$ and $\mathfrak{z}_{\mathfrak{g}}\left(\mathfrak{c}_{11}\right)_{11}=\mathfrak{c}_{11}$. Our idea is to replace $\mathfrak{c}_{11}$ with a proper subspace $\tilde{\mathfrak{c}}$ such that 
Then we consider $\hat{\mathfrak{c}}:=\mathfrak{z}_{\mathfrak{g}}(\tilde{\mathfrak{c}})_{10} \times \tilde{\mathfrak{c}} \subset \mathfrak{E}$ and compute the dimension of $G_{00} \cdot \hat{\mathfrak{c}}$. Since $\overline{G_{00} \cdot \mathfrak{c}_{11}}=\mathfrak{g}_{11}$, we have

$$
\operatorname{dim} G_{00}+\operatorname{dim} \mathfrak{c}_{11}-\operatorname{dim} \mathfrak{z}_{\mathfrak{g}}\left(\mathfrak{c}_{11}\right)_{00}=\operatorname{dim} \mathfrak{g}_{11} .
$$

Set $\mathfrak{T}_{00}(\hat{\mathfrak{c}})=\left\{g \in G_{00} \mid g \cdot y \in \hat{\mathfrak{c}}\right.$ for generic $\left.y \in \hat{\mathfrak{c}}\right\}$, and likewise for $\mathfrak{c}_{11}$. In view of $(5-1)$, we have $\operatorname{dim} \mathfrak{T}_{00}(\hat{\mathfrak{c}})=\operatorname{dim} \mathfrak{T} T_{00}\left(\mathfrak{c}_{11}\right)=\operatorname{dim} \mathfrak{z}_{\mathfrak{g}}\left(\mathfrak{c}_{11}\right)_{00}$. Then

$$
\begin{aligned}
\operatorname{dim} G_{00} \cdot \hat{\mathfrak{c}} & =\operatorname{dim} G_{00}+\operatorname{dim} \hat{\mathfrak{c}}-\operatorname{dim} \mathfrak{T}_{00}(\hat{\mathfrak{c}}) \\
& =\left(\operatorname{dim} G_{00}+\operatorname{dim} \mathfrak{c}_{11}-\operatorname{dim} \mathfrak{z}_{\mathfrak{g}}\left(\mathfrak{c}_{11}\right)_{00}\right)+\left(\operatorname{dim} \mathfrak{z}_{\mathfrak{g}}(\tilde{\mathfrak{c}})_{10}-\operatorname{dim} \mathfrak{c}_{11}+\operatorname{dim} \tilde{\mathfrak{c}}\right) \\
& =\operatorname{dim} \mathfrak{g}_{11}+\left(\operatorname{dim} \hat{\mathfrak{c}}-\operatorname{dim} \mathfrak{c}_{11}\right) .
\end{aligned}
$$

Thus, we obtain a subvariety of larger dimension, if $\operatorname{dim} \mathfrak{z}_{\mathfrak{g}}(\tilde{\mathfrak{c}})_{10}+\operatorname{dim} \tilde{\mathfrak{c}}>\operatorname{dim} \mathfrak{c}_{11}$. Of course, it is not always possible to construct such a $\tilde{\mathfrak{c}}$. Our sufficient condition exploits restricted root systems. Set $\mathfrak{h}=\mathfrak{g}^{\sigma_{3}}$, and let $H$ denote the corresponding connected (reductive) subgroup of $G$. Write $\bar{\sigma}$ for the restriction to $H$ of $\sigma_{1}$ or $\sigma_{2}$.

Let $C_{11}=\exp \left(\mathfrak{c}_{11}\right) \subset H \subset G$ be the corresponding torus. The coincidence of CSS means that $C_{11}$ is a maximal $\sigma_{1}$-anisotropic torus in $G$ and a maximal $\bar{\sigma}$-anisotropic torus in $H$. Accordingly, one obtains the inclusion of two restricted root systems relative to $C_{11}$ :

$$
\Psi\left(H / G_{00}\right) \subset \Psi\left(G / G_{0 \star}\right) .
$$

Identifying restricted roots and their differentials, one may consider restricted roots as linear forms on $\mathfrak{c}_{11}$. Then the set of $G_{00}$-regular elements of $\mathfrak{c}_{11}$ is

$$
\left\{x \in \mathfrak{c}_{11} \mid \mu(x) \neq 0 \text { for all } \mu \in \Psi\left(H / G_{00}\right)\right\}
$$

and the set of $G_{0 \star}$-regular elements is $\left\{x \in \mathfrak{c}_{11} \mid \mu(x) \neq 0\right.$ for all $\left.\mu \in \Psi\left(G / G_{0 \star}\right)\right\}$.

Proposition 5.1. Assume that $\mu \in \Psi\left(G / G_{0 \star}\right)$ and $r \mu \notin \Psi\left(H / G_{00}\right)$ for any $r \in \mathbb{Q}$. If $m_{\mu}>1$, then $\operatorname{dim} \mathfrak{E} \geqslant \operatorname{dim} \mathfrak{g}_{11}+m_{\mu}-1>\operatorname{dim} \mathfrak{g}_{11}$.

Proof. Under this assumption, $\tilde{\mathfrak{c}}:=\operatorname{Ker}(\mu) \subset \mathfrak{c}_{11}$ still contains $G_{00}$-regular elements, and $\operatorname{dim} \tilde{\mathfrak{c}}=\operatorname{dim} \mathfrak{c}_{11}-1$. Furthermore, $\mathfrak{z}_{\mathfrak{g}}(\tilde{\mathfrak{c}})$ is $\overrightarrow{\boldsymbol{\sigma}}$-stable and $\mathfrak{z}_{\mathfrak{g}}(\tilde{\mathfrak{c}})=\mathfrak{z}_{\mathfrak{g}}\left(\mathfrak{c}_{11}\right) \oplus \mathfrak{g}_{\mu} \oplus \mathfrak{g}_{-\mu}$. Recall that $\mathfrak{z}_{\mathfrak{g}}\left(\mathfrak{c}_{11}\right)$ is contained in $\mathfrak{g}_{00} \oplus \mathfrak{g}_{11}$. Clearly, $\mathfrak{g}_{\mu} \oplus \mathfrak{g}_{-\mu}$ is also $\overrightarrow{\boldsymbol{\sigma}}$-stable and is contained in $\mathfrak{g}_{01} \oplus \mathfrak{g}_{10}$.

Since $\left\{\sigma_{1}, \sigma_{2}\right\}$ is a dyad, $\operatorname{dim}\left(\mathfrak{g}_{\mu} \oplus \mathfrak{g}_{-\mu}\right) \cap \mathfrak{g}_{10}=\operatorname{dim}\left(\mathfrak{g}_{\mu} \oplus \mathfrak{g}_{-\mu}\right) \cap \mathfrak{g}_{01}=m_{\mu}$. Hence $\operatorname{dim} \mathfrak{z}_{\mathfrak{g}}(\tilde{\mathfrak{c}})_{10}=m_{\mu}$, and the assertion follows from (5-2).

Remark 5.2. (1) Such a construction gives nothing, if all root multiplicities in $\Psi\left(G / G_{0 \star}\right)$ are equal to 1 . For instance, if $\sigma_{1}$ is of maximal rank.

(2) The procedure described in the previous proof admits obvious modifications. Roughly speaking, if there are linearly independent roots $\mu_{1}, \mu_{2}, \ldots$, in $\Psi\left(G / G_{0 \star}\right)$, with large multiplicities, such that $\mathbb{Q}-\operatorname{span}\left\{\mu_{1}, \mu_{2}, \ldots\right\} \cap \Psi\left(H / G_{00}\right)=\varnothing$, then one can take $\tilde{\mathfrak{c}}=\operatorname{Ker}\left(\mu_{1}, \mu_{2}, \ldots\right)$, see Proposition 6.5. 
Although it is convenient to stick to one specific $\overrightarrow{\boldsymbol{\sigma}}$-commuting variety in theoretical considerations, it may happen that in concrete examples different $\overrightarrow{\boldsymbol{\sigma}}$-commuting varieties exhibit different good (or bad) properties.

Example 5.3. Let $\sigma_{1}$ be an outer involution of $\mathfrak{g}=\mathfrak{s l}_{2 n}$ with $\mathfrak{g}^{\sigma_{1}}=\mathfrak{s p}_{2 n}$. In [Panyushev 2013, §2], we gave a method for describing all the dyads including $\sigma_{1}$, which exploits the restricted root system $\Psi\left(G / G^{\sigma_{1}}\right)$. This implies that one can find $\sigma_{2}$ conjugated $\sigma_{1}$ such that the inner involution $\sigma_{3}=\sigma_{1} \sigma_{2}$ has the fixed-point subalgebra $\mathfrak{h}=\mathfrak{s l}_{2 m} \oplus \mathfrak{s l}_{2 n-2 m} \oplus \mathfrak{t}_{1}$. The corresponding quaternionic decomposition is

$$
\mathfrak{s l}_{2 n}=\begin{array}{c:c}
\mathfrak{s p}_{2 m} \oplus \mathfrak{s p}_{2 n-2 m} & \mathrm{R}\left(\varpi_{1}\right) \mathrm{R}\left(\varpi_{1}^{\prime}\right) \\
\mathrm{R}\left(\varpi_{1}\right) \mathrm{R}\left(\varpi_{1}^{\prime}\right) & \mathrm{R}\left(\varpi_{2}\right)+\mathrm{R}\left(\varpi_{2}^{\prime}\right)+\mathrm{R}(0)
\end{array} \sigma_{1},
$$

$\sigma_{2}$

where $\varpi_{i}$ (resp. $\varpi_{i}^{\prime}$ ) are fundamental weights of $\mathfrak{s p}_{2 m}$ (resp. $\mathfrak{s p}_{2 n-2 m}$ ), and $\mathrm{R}(\lambda)$ is a simple module of the respective simple Lie algebra with highest weight $\lambda$.

- Here $G=\mathrm{SL}_{2 n}, G_{0 \star}=\mathrm{Sp}_{2 n}, H=\mathrm{SL}_{2 m} \times \mathrm{SL}_{2(n-m)} \times T_{1}$, and $G_{00}=S p_{2 m} \times$ $S p_{2(n-m)}$. According to [Helgason 1978, Chapter X, Table VI], we have $\Psi\left(G / G_{0 \star}\right)$ $=\mathbf{A}_{n-1}, \Psi\left(H / G_{00}\right)=\mathbf{A}_{m-1}+\mathbf{A}_{n-m-1}$, and all root multiplicities in $\Psi\left(G / G_{0 \star}\right)$ equal 4. Since $\Psi\left(H / G_{00}\right)$ has fewer roots, Proposition 5.1 implies that $\mathfrak{E}$ has an irreducible component of dimension greater than $\operatorname{dim} \mathfrak{g}_{11}+(4-1)$ and our test map $\varphi: \mathfrak{g}_{10} \times \mathfrak{g}_{11} \rightarrow \mathfrak{g}_{01}$ is not equidimensional.

- Here $\operatorname{dim} \mathfrak{c}_{01}=\operatorname{dim} \mathfrak{c}_{10}=\min \{m, n-m\}$ and any big CSS in $\mathfrak{g}_{10} \oplus \mathfrak{g}_{01}$ is of dimension $2 \min \{m, n-m\}$. By Theorem 3.7(2), this means that all homogeneous CSS in $\mathfrak{g}_{10} \oplus \mathfrak{g}_{01}$ are $G_{00}$-conjugate, and therefore the $\overrightarrow{\boldsymbol{\sigma}}$-commuting variety related to the commutator $\mathfrak{g}_{10} \oplus \mathfrak{g}_{01} \rightarrow \mathfrak{g}_{11}$ has a unique standard component.

Example 5.4. Let $\sigma$ be an involution of $\mathfrak{g}=\mathbf{E}_{7}$ with $\mathfrak{g}^{\sigma}=\mathbf{D}_{6} \times \mathbf{A}_{1}$. It can be included in two nonconjugate triads [Kollross 2009]. One of them has $\mathfrak{g}_{00}=\mathbf{D}_{4} \times \mathbf{A}_{1}^{3}$, with quaternionic decomposition

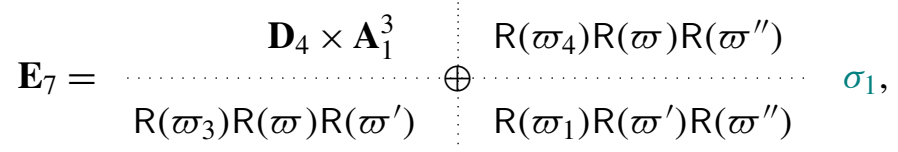

$\sigma_{2}$

where $\varpi, \varpi^{\prime}$, and $\varpi^{\prime \prime}$ are the fundamental weights of the simple factors of $\mathbf{A}_{1}^{3}$, and $\varpi_{i}$ are fundamental weights of $\mathbf{D}_{4}$. Here $\operatorname{dim} \mathfrak{g}_{i j}=32$ for $(i j) \neq(00)$ and our test commutator map is

$$
\varphi: \mathrm{R}\left(\varpi_{3}\right) \mathrm{R}(\varpi) \mathrm{R}\left(\varpi^{\prime}\right) \times \mathrm{R}\left(\varpi_{1}\right) \mathrm{R}\left(\varpi^{\prime}\right) \mathrm{R}\left(\varpi^{\prime \prime}\right) \rightarrow \mathrm{R}\left(\varpi_{4}\right) \mathrm{R}(\varpi) \mathrm{R}\left(\varpi^{\prime \prime}\right) .
$$


Using [Helgason 1978, Chapter X, Table VI], we find that $r k\left(\mathbf{E}_{7} / \mathbf{D}_{6} \times \mathbf{A}_{1}\right)=4$ and the restricted root system $\Psi\left(\mathbf{E}_{7} / \mathbf{D}_{6} \times \mathbf{A}_{1}\right)$ is of type $\mathbf{F}_{4}$; whereas $r k\left(\mathbf{D}_{6} \times\right.$ $\left.\mathbf{A}_{1} / \mathbf{D}_{4} \times \mathbf{A}_{1}^{3}\right)=\operatorname{rk}\left(\mathbf{D}_{6} / \mathbf{D}_{4} \times \mathbf{A}_{1}^{2}\right)=4$ and the corresponding root system is of type $\mathbf{B}_{4}$. The long (resp. short) roots of $\mathbf{B}_{4}$ are also long (resp. short) roots of $\mathbf{F}_{4}$, and the multiplicities are $m_{\text {long }}=1$ and $m_{\text {short }}=4$. However, the root system $\mathbf{B}_{4}$ has fewer short roots than $\mathbf{F}_{4}$. Therefore, Proposition 5.1 applies here, and $\mathfrak{E}$ has an irreducible component of dimension at least $\mathfrak{m}_{\text {short }}-1+\operatorname{dim} \mathfrak{g}_{11}=35$.

Example 5.5. Let $\sigma$ be an involution of $\mathfrak{g}=\mathbf{F}_{4}$ with $\mathfrak{g}^{\sigma}=\mathbf{B}_{4}=\mathfrak{s o}_{9}$. Up to conjugacy, this involution can be included in a unique triad [Kollross 2009], with quaternionic decomposition

$$
\mathbf{F}_{4}=\begin{array}{l:l}
\mathbf{D}_{4} & \mathrm{R}\left(\varpi_{4}\right) \\
\hdashline \mathrm{R}\left(\varpi_{3}\right) & \mathrm{R}\left(\varpi_{1}\right)
\end{array} \sigma_{1},
$$

where $\operatorname{dim} \mathrm{R}\left(\varpi_{i}\right)=8$ and the main diagonal represents the little involution of $\mathfrak{g}^{\sigma_{3}}=\mathbf{B}_{4}=\mathfrak{s o}_{9}$. Our test commutator is the bilinear $\mathbf{D}_{4}$-equivariant mapping $\mathrm{R}\left(\varpi_{3}\right) \times \mathrm{R}\left(\varpi_{1}\right) \rightarrow \mathrm{R}\left(\varpi_{4}\right)$. Here $\operatorname{rk}\left(\mathbf{F}_{4} / \mathbf{B}_{4}\right)=1$ and the restricted root system $\Psi\left(\mathbf{F}_{4} / \mathbf{B}_{4}\right)$ is of type $\mathbf{B C}_{1}$. The restricted root system $\Psi\left(\mathbf{B}_{4} / \mathbf{D}_{4}\right)$ is of type $\mathbf{C}_{1}$. Since all little and big CSS are one-dimensional, Proposition 5.1 does not help here. Actually, the only standard components of $\mathfrak{E}$ are $\mathfrak{g}_{10} \times\{0\}$ and $\{0\} \times \mathfrak{g}_{11}$, both of dimension eight. Below, we describe an "intermediate" nonstandard irreducible component of dimension eleven.

Let $x \in \mathfrak{g}_{11} \simeq \mathrm{R}\left(\varpi_{1}\right)$ be a nonzero nilpotent element. All such elements form a sole seven-dimensional $\mathrm{SO}_{8}$-orbit. By Lemma $1.1, \operatorname{dim~} \mathrm{SO}_{9} \cdot x=2 \cdot 7=14$ and hence $\operatorname{dim}\left(\mathfrak{s o}_{9}\right)^{x}=22$. The only nilpotent $\mathrm{SO}_{9}$-orbit of dimension fourteen in $\mathfrak{s o}_{9}$ is the orbit of short root vectors. The short roots of $\mathfrak{g}^{\sigma_{3}}=\mathbf{B}_{4}$ are also short roots of $\mathfrak{g}=\mathbf{F}_{4}$. Therefore, a minimal including regular semisimple subalgebra is $\tilde{\mathbf{A}}_{1}$ in Dynkin's notation. This implies that $\operatorname{dim}_{\mathfrak{z} g}(x)=30$ and completely determines the dimension matrix of the spaces $\mathfrak{z}_{\mathfrak{g}}(x)_{i j}$ :

$$
\begin{array}{l|l}
21 & 4 \\
\hline 4 & 1
\end{array} .
$$

Here the one-dimensional space $\mathfrak{g}_{11}$ is just the line $\mathbb{k} x$. Then $\operatorname{dim} \overline{G_{00} \cdot\left(\mathfrak{z}_{\mathfrak{g}}(x)_{10} \oplus \mathbb{k} x\right)}$ $=4+7=11$. Using the projection $\mathfrak{E} \rightarrow \mathfrak{g}_{11}$, one can prove that $\overline{G_{00} \cdot\left(\mathfrak{z}_{\mathfrak{g}}(x)_{10} \oplus \mathbb{k} x\right)}$ is the only new irreducible component of $\mathfrak{E}$. It is contained in $\mathcal{N}_{10} \times \mathcal{N}_{11}$. Thus, $\mathfrak{E}$ has three irreducible components.

\section{Triads of Hermitian involutions and simple Jordan algebras}

In this section, $\mathfrak{g}$ is assumed to be simple. We say that $\sigma \in \operatorname{lnv}(\mathfrak{g})$ is Hermitian if $\mathfrak{g}_{0}$ is not semisimple. All these involutions are associated with $\mathbb{Z}$-gradings of $\mathfrak{g}$ with 
only three nonzero terms (short gradings), that is, with parabolic subalgebras with abelian nilpotent radical. Let $\mathfrak{g}=\mathfrak{g}(-1) \oplus \mathfrak{g}(0) \oplus \mathfrak{g}(1)$ be a short grading. Then $\mathfrak{p}=$ $\mathfrak{g}(0) \oplus \mathfrak{g}(1)$ is a (maximal) parabolic subalgebra with abelian nilpotent radical, and one defines a Hermitian involution $\sigma$ by letting $\mathfrak{g}^{\sigma}=\mathfrak{g}(0)$ and $\mathfrak{g}_{1}^{(\sigma)}=\mathfrak{g}(-1) \oplus \mathfrak{g}(1)$.

Since $\mathfrak{g}$ is simple, the center of $\mathfrak{g}(0)$ is one-dimensional and there is a unique $h \in \mathfrak{g}(0)$ such that $\mathfrak{g}(i)=\{x \in \mathfrak{g} \mid[h, x]=2 i x\}$. By [Vinberg 1976, §2.3], the reductive group $G(0)$ has finitely many orbits in $\mathfrak{g}(1)$. Let $\mathcal{O}$ be the dense $G(0)$-orbit in $\mathfrak{g}(1)$ and $e \in \mathcal{O}$. Set $\mathfrak{g}(i)^{e}=\mathfrak{g}(i) \cap \mathfrak{g}^{e}$.

For future reference, we provide a proof of the following well-known assertion.

Lemma 6.1. $h \in[\mathfrak{g}, e] \Longleftrightarrow \mathfrak{g}(0)^{e}$ is reductive.

Proof. (1) If $h \in[\mathfrak{g}, e]$, then $h=[e, f]$ for some $f \in \mathfrak{g}(-1)$ and therefore, $\{e, h, f\}$ is an $\mathfrak{s l}_{2}$-triple. Then $\mathfrak{g}(0)^{e}=\mathfrak{z}_{\mathfrak{g}}(e, h, f)$, which is reductive.

(2) For $e \in \mathcal{O}$, we have $\operatorname{dim} \mathfrak{g}(0)^{e}=\operatorname{dim} \mathfrak{g}(0)-\operatorname{dim} \mathfrak{g}(1)$. Using the Kirillov-Kostant form associated with $e$, we see that $\operatorname{dim} \mathfrak{g}(-1)-\operatorname{dim} \mathfrak{g}(-1)^{e}=\operatorname{dim} \mathfrak{g}(0)-\operatorname{dim} \mathfrak{g}(0)^{e}$. Hence $\mathfrak{g}(-1)^{e}=0$ and $\mathfrak{g}^{e}=\mathfrak{g}(0)^{e} \oplus \mathfrak{g}(1)$. Set $\mathfrak{k}=\mathfrak{g}(0)^{e}$, and let ()$^{\perp}$ denote the orthocomplement with respect to the Killing form. Then $[\mathfrak{g}, e]=\left(\mathfrak{g}^{e}\right)^{\perp}=$ $\mathfrak{g}(1) \oplus\left(\mathfrak{k}^{\perp} \cap \mathfrak{g}(0)\right)$. Now, if $\mathfrak{k}$ is reductive, then the restriction of the Killing form to $\mathfrak{k}$ is nondegenerate and $\mathfrak{m}:=\mathfrak{k}^{\perp} \cap \mathfrak{g}(0)$ is a $\mathfrak{k}$-stable complement to $\mathfrak{k}$ in $\mathfrak{g}(0)$. Since $\operatorname{dim}[\mathfrak{g}(-1), e]=\operatorname{dim} \mathfrak{g}(1)=\operatorname{dim} \mathfrak{g}(0)-\operatorname{dim} \mathfrak{k}$, we conclude that $\mathfrak{m}=[\mathfrak{g}(-1), e]$. Thus, $e$ acts on $\mathfrak{g}$ as follows:

$$
\left\{\begin{array}{c}
\mathfrak{g}(-1) \stackrel{\sim}{\longrightarrow} \mathfrak{m} \stackrel{\sim}{\longrightarrow} \mathfrak{g}(1) \rightarrow 0 \\
\mathfrak{k} \rightarrow 0 .
\end{array}\right.
$$

Let $\{e, \tilde{h}, f\}$ be an $\mathfrak{s l}_{2}$-triple with $\tilde{h} \in \mathfrak{g}(0)$ and $f \in \mathfrak{g}(-1)$. Such a triple always exists, see [Vinberg 1979, §2]. Then (6-1) shows that $\mathfrak{g}$ is a sum of three-dimensional and one-dimensional $\mathfrak{s l}_{2}$-modules, and that $\mathfrak{g}^{\tilde{h}}=\mathfrak{k} \oplus \mathfrak{m}$. Since $\mathfrak{g}(0)$ has a one-dimensional center, one must have $\tilde{h}=h$. Thus, $h \in[\mathfrak{g}, e]$.

Theorem 6.2. Suppose that a Hermitian involution $\sigma=\sigma_{1}$ has the property that $\mathfrak{g}(0)^{e}$ is reductive. Then $\sigma_{1}$ can be included in a triad.

Proof. Using the notation of the previous proof, we set $\mathfrak{k}=\mathfrak{g}(0)^{e}$ and take (the unique) $f \in \mathfrak{g}(-1)$ such that $h=[e, f]$. Then $\{e, h, f\}$ is an $\mathfrak{s l}_{2}$-triple, $[e, \mathfrak{g}(-1)]=: \mathfrak{m}$ is a complementary $\mathfrak{k}$-submodule to $\mathfrak{k}$ in $\mathfrak{g}(0)$, and $[e,[e, \mathfrak{g}(-1)]]=\mathfrak{g}(1)$. This also shows that $\mathfrak{g}(-1), \mathfrak{m}$, and $\mathfrak{g}(1)$ are isomorphic $\mathfrak{k}$-modules.

In this case, $\mathfrak{k}$ is the fixed-point subalgebra of an involution of $\mathfrak{g}(0)$ and for this involution the (-1)-eigenspace is $\mathfrak{m}$ (see [Panyushev 1994a, proof of Proposition 3.3]). Let $\sigma_{2}$ denote this involution of $\mathfrak{g}(0)$. Then $\sigma_{2}(h)=-h$. We extend $\sigma_{2}$ to the whole of $\mathfrak{g}$ by letting $\sigma_{2}(e)=f$. Then $\sigma_{2}([x, e])=[-x, f]$ for all $x \in \mathfrak{m}$, which defines $\sigma_{2}$ on $\mathfrak{g}(1)$ and shows that $\sigma_{2}(\mathfrak{g}(1)) \subset \mathfrak{g}(-1)$. Clearly, $\sigma_{1}$ and $\sigma_{2}$ commute. Furthermore, $\sigma_{1}$ and $\sigma_{2}$ are different involutions of the three-dimensional simple 
subalgebra $\langle e, h, f\rangle$. This implies that $\sigma_{1}, \sigma_{2}$, and $\sigma_{3}=\sigma_{1} \sigma_{2}$ are already conjugate with respect to $\mathrm{PSL}_{2}=$ Aut $\langle e, h, f\rangle$. In particular, $\left\{\sigma_{1}, \sigma_{2}, \sigma_{3}\right\}$ is a triad.

This theorem can be derived from the classification of triads, but our direct construction allows us to visualize the resulting quaternionic decomposition rather explicitly. We have

$$
\mathfrak{g}=\begin{array}{c:c}
\mathfrak{k} & \mathfrak{m} \\
\hdashline \mathfrak{m}, e-f] & \oplus \ldots \ldots \ldots \ldots \ldots \ldots \ldots
\end{array} \sigma_{1}
$$

Here $h \in \mathfrak{m}=\mathfrak{g}_{01}, e+f \in[\mathfrak{m}, e-f]=\mathfrak{g}_{10}$, and $e-f \in[\mathfrak{m}, e+f]=\mathfrak{g}_{11}$. Note also that $\mathfrak{k} \oplus \mathfrak{m}=\mathfrak{g}(0)$ and $[\mathfrak{m}, e-f] \oplus[\mathfrak{m}, e+f]=\mathfrak{g}(1) \oplus \mathfrak{g}(-1)$.

Remark. If $\mathfrak{g}(0)^{e}$ is not reductive, then such a triad may not exist. For instance, if $\mathfrak{g}=\mathfrak{s l}_{2 n}$ and $\mathfrak{g}_{0}=\mathfrak{s l}_{m} \times \mathfrak{s l}_{2 n-m} \times \mathfrak{t}_{1}$ with $n \neq m$ and $m$ odd, then there is no respective triad, see [Vinberg 2005, 3.2].

As is well known, if $\mathfrak{g}(0)^{e}$ is reductive, then $\mathfrak{g}(-1)$ has a structure of a simple Jordan algebra. Namely, for $x, y \in \mathfrak{g}(-1)$, we set

$$
x \circ y=[x,[e, y]] \in \mathfrak{g}(-1) .
$$

Then $\{\mathfrak{g}(-1), \circ\}$ is a simple Jordan algebra [Tits 1962; Kantor 1964]. (See also [Kac 1980, §4] for possible generalizations). Here $\mathfrak{k}=\mathfrak{g}_{00}$ is the Lie algebra of derivations of $\{\mathfrak{g}(-1), \circ\}$. The triad constructed in Theorem 6.2 is called a Jordan triad.

Definition 3. The commuting variety of a Jordan algebra $\{\mathcal{J}, \circ\}$ is

$$
\mathfrak{E}(\mathcal{J})=\{(x, y) \mid x \circ y=0\} \subset \mathcal{J} \times \mathcal{J} .
$$

The Jordan triad (6-2) provides a link between the commutator mapping $\varphi$ : $\mathfrak{g}_{10} \times \mathfrak{g}_{11} \rightarrow \mathfrak{g}_{01}$ and the commuting variety of the simple Jordan algebra $\mathfrak{g}(-1)$.

Theorem 6.3. The commuting variety of the Jordan algebra $\{\mathfrak{g}(-1)$, o $\}$ is isomorphic to the zero fiber of the commutator mapping $\varphi: \mathfrak{g}_{10} \times \mathfrak{g}_{11}=[\mathfrak{m}, e-f] \times$ $[\mathfrak{m}, e+f] \rightarrow \mathfrak{m}=\mathfrak{g}_{01}$.

Proof. Any element of $\mathfrak{m}$ can uniquely be written as $[x, e]$ with $x \in \mathfrak{g}(-1)$. So, if $[x, e],[y, e] \in \mathfrak{m}$ are arbitrary, then $[[x, e], e-f] \in \mathfrak{g}_{10}$ and $[[y, e], e+f] \in \mathfrak{g}_{11}$ are arbitrary and $\varphi$ takes the corresponding pair to $[[[x, e], e-f],[[y, e], e+f]] \in$ $\mathfrak{m}=\mathfrak{g}_{01}$. It is a good exercise in the Jacobi identity to check that

$$
[[[x, e], e-f],[[y, e], e+f]]=2[[[x, e], y], e] .
$$


(One should use the fact that $h=[e, f]$ is the defining element of the short grading. Hence $[[x, e], f]=2 x$, etc.) Since $a=[[x, e], y] \in \mathfrak{g}(-1)$ and $\mathfrak{g}^{e} \cap \mathfrak{g}(-1)=0$, we have $[a, e]=0$ if and only if $a=0$. Therefore,

$$
\begin{aligned}
([[x, e], e-f],[[y, e], e+f]) \in \varphi^{-1}(0) & \Longleftrightarrow[[x, e], y]=0 \\
& \Longleftrightarrow(x, y) \in \mathfrak{E}(\mathfrak{g}(-1)) .
\end{aligned}
$$

If $\mathcal{J}$ is a simple Jordan algebra, then the operator $L_{x}: \mathcal{J} \rightarrow \mathcal{J}, L_{x}(y)=x \circ y$, is invertible for almost all $x$. Therefore, $\mathcal{J} \times\{0\}$ and $\{0\} \times \mathcal{J}$ are two irreducible components of $\mathfrak{E}(\mathcal{J})$. Clearly, there are some other irreducible components. It is an interesting problem to determine all the components of $\mathfrak{E}(\mathcal{J})$ and their dimensions.

The list of Hermitian involutions leading to Jordan triads and simple Jordan algebras is given in Table 1 . We point out the semisimple subalgebra $\mathfrak{s}=[\mathfrak{g}(0), \mathfrak{g}(0)]$ and the structure of $\mathfrak{g}(1)$ as a $\mathfrak{s}$-module. Here the $\varpi_{i}$ are the fundamental weights of $\mathfrak{s}$.

Remark. The Jordan multiplication in the space $\mathrm{Skew}_{2 n}$ of usual skew-symmetric matrices is defined as follows. If $A, B, J \in \mathrm{Skew}_{2 n}$ and $J$ is nondegenerate, then $A \circ B=\frac{1}{2}(A J B+B J A)$.

There are some coincidences for small $n$. Namely,

$$
\text { Item } 1(n=1) \simeq \operatorname{Item} 2(n=1), \quad \text { Item } 1(n=2) \simeq \text { Item } 4(n=3) .
$$

Furthermore, if $n=1$ in Item 3 , then $\mathfrak{g}$ is not simple. This explains the conditions on $n$ given in the last column. For Item 2, the Hermitian involution (of $\mathfrak{s p}_{2 n}$ ) is of maximal rank and the respective Jordan algebra is the algebra Sym ${ }_{n}$ of symmetric $n \times n$ matrices. Therefore, by Theorems 4.1 and 6.3, the multiplication morphism $\circ: \operatorname{Sym}_{n} \times \operatorname{Sym}_{n} \rightarrow \operatorname{Sym}_{n}$ is equidimensional, that is, $\operatorname{dim} \mathfrak{E}\left(\operatorname{Sym}_{n}\right)=\operatorname{dim} \operatorname{Sym}_{n}=$ $\left(n^{2}+n\right) / 2$.

In all other cases, the multiplication morphism $\mathcal{J} \times \mathcal{J} \rightarrow \mathcal{J}$ is not equidimensional, see Proposition 6.5. Before checking this, we give an "elementary" explanation for the Jordan algebra of all matrices (Item 1).

\begin{tabular}{c|ccccc|c} 
& $\mathfrak{g}$ & $\mathfrak{s}$ & $\mathfrak{g}(1)$ & $\mathfrak{k}$ & $\mathcal{J}$ & \\
\hline 1 & $\mathfrak{s l}_{2 n}$ & $\mathfrak{s l}_{n} \oplus \mathfrak{s l}_{n}$ & $\mathrm{R}\left(\varpi_{1}\right) \otimes \mathrm{R}\left(\varpi_{1}^{\prime}\right)$ & $\mathfrak{s l}_{n}$ & $n \times n$ matrices & $n \geqslant 1$ \\
2 & $\mathfrak{s p}_{2 n}$ & $\mathfrak{s l}_{n}$ & $\mathrm{R}\left(2 \varpi_{1}\right)$ & $\mathfrak{s o}_{n}$ & symmetric $n \times n$ matrices & $n \geqslant 2$ \\
3 & $\mathfrak{s o}_{4 n}$ & $\mathfrak{s l}_{2 n}$ & $\mathrm{R}\left(\varpi_{2}\right)$ & $\mathfrak{s p}_{2 n}$ & skew-symm. $2 n \times 2 n$ matrices & $n \geqslant 2$ \\
4 & $\mathfrak{s o}_{n+2}$ & $\mathfrak{s o}_{n}$ & $\mathrm{R}\left(\varpi_{1}\right)$ & $\mathfrak{s o}_{n-1}$ & spin-factor & $n \geqslant 4$ \\
5 & $\mathbf{E}_{7}$ & $\mathbf{E}_{6}$ & $\mathrm{R}\left(\varpi_{1}\right)$ & $\mathbf{F}_{4}$ & the Albert algebra & \\
\hline
\end{tabular}

Table 1. List of Hermitian involutions leading to Jordan triads and simple Jordan algebras. 
Example 6.4. Let $M$ be the associative (and also Lie and Jordan) algebra of all $n \times n$ matrices. That is, we exploit the usual matrix product, the Lie bracket $[A, B]=A B-B A$, and the Jordan product $A \circ B=(A B+B A) / 2$. Let $\chi(B)=$ $\operatorname{det}(\lambda I-B)=\sum_{i} \chi_{n-i}(B) \lambda^{i}$ be the characteristic polynomial of a matrix $B$. Let $\mathfrak{z}^{\mathrm{J}}(B)$ and $\mathfrak{z}^{\mathrm{Lie}}(B)$ denote the Jordan and Lie centralizers of $B$, respectively. Consider the subvariety

$$
\mathrm{M}^{\langle 2\rangle}=\left\{B \in \mathrm{M} \mid \chi_{2 i+1}(B)=0 \text { for all } i\right\} .
$$

It is an irreducible complete intersection and codim $\mathrm{M}^{\langle 2\rangle}=[n+1 / 2]$ (see [Richardson 1987, Lemma 5.3]). We also need the dense open subset $M^{\text {reg }}$ of regular elements (in the Lie algebra sense) and the subvariety

$$
\mathrm{M}^{\mathrm{ev}}=\{B \in \mathrm{M} \mid B \text { is conjugate to }-B\} .
$$

If $B \in \mathrm{M}^{\mathrm{ev}}$ and $A B A^{-1}=-B$, then $A \in \mathfrak{z}^{J}(B)$ and the mapping $C \in \mathfrak{z}^{\text {Lie }}(B) \mapsto$ $A C \in \mathfrak{z}^{\mathrm{J}}(B)$ is a linear isomorphism. In particular, $\operatorname{dim} \mathfrak{z}^{\mathrm{J}}(B)=\operatorname{dim} \mathfrak{z}^{\text {Lie }}(B)$. The following is clear:

- $\mathrm{M}^{\langle 2\rangle} \cap \mathrm{M}^{\text {reg }} \neq \varnothing$ (it contains a regular nilpotent element).

- $\mathrm{M}^{\mathrm{ev}} \subset \mathrm{M}^{\langle 2\rangle}$ and $\mathrm{M}^{\mathrm{ev}} \cap \mathrm{M}^{\mathrm{reg}} \neq \varnothing$.

Claim. We have $\mathrm{M}^{\langle 2\rangle} \cap \mathrm{M}^{\text {reg }} \subset \mathrm{M}^{\mathrm{ev}}$. In particular, $\operatorname{dim} \mathfrak{z}^{J}(B)=n$ for almost all $B \in \mathrm{M}^{\langle 2\rangle}$.

Proof. If $B \in \mathrm{M}^{\langle 2\rangle} \cap \mathrm{M}^{\text {reg }}$, then $B$ and $-B$ are both regular and have the same Jordan blocks and the same eigenvalues. Hence $B$ and $-B$ are conjugate.

Let $\mathfrak{E}^{J}(\mathrm{M})$ denote the Jordan commuting variety and $p: \mathfrak{E}^{J}(\mathrm{M}) \rightarrow \mathrm{M}$ the projection to the first factor. The previous analysis implies that

$$
\operatorname{dim} p^{-1}\left(\mathrm{M}^{\langle 2\rangle} \cap \mathrm{M}^{\mathrm{reg}}\right)=\operatorname{dim} \mathrm{M}^{\langle 2\rangle}+n=n^{2}+[n / 2] .
$$

Thus, $\operatorname{dim} \mathfrak{E}^{J}(\mathrm{M}) \geqslant n^{2}+[n / 2]>\operatorname{dim} \mathrm{M}$. One can prove that this yields an irreducible component of maximal dimension; that is, $\operatorname{dim} \mathfrak{E}^{J}(\mathrm{M})=n^{2}+[n / 2]$.

Table 2 contains information on the restricted root systems associated with Jordan triads. For a Hermitian involution $\sigma$, we point out Lie algebras $\mathfrak{g}, \mathfrak{h}=\mathfrak{g}^{\sigma}, \mathfrak{g}_{00}=\mathfrak{k}$, the restricted root systems $\Psi(G / H)$ and $\Psi\left(H / G_{00}\right)$, and the multiplicity of the short roots in $\Psi(G / H)$, denoted $m_{\text {short }}$. For all items in Table 2, the multiplicity of long roots in $\Psi(G / H)$ equals 1 and $\Psi\left(H / G_{00}\right)$ is embedded in $\Psi(G / H)$ as a subset of short roots.

The root system of type $\mathbf{C}_{n}$ has some short roots that are not roots of $\mathbf{A}_{n-1}$. Therefore, Proposition 5.1 guarantees the existence of a subvariety in $\mathfrak{E}(\mathcal{J})$ of dimension $\operatorname{dim} \mathcal{J}+m_{\text {short }}-1$, which is larger than the dimension of a generic fiber if $m_{\text {short }}>1$. However, a clever choice of $\tilde{\mathfrak{c}} \subset \mathfrak{c}_{11}$ (see Remark 5.2(2)) allows us to get a better lower bound on $\operatorname{dim} \mathfrak{E}(\mathcal{J})$ : 


\begin{tabular}{c|cccccc} 
& $\mathfrak{g}$ & $\mathfrak{h}$ & $\mathfrak{g}_{00}$ & $\Psi(G / H)$ & $m_{\text {short }}$ & $\Psi\left(H / G_{00}\right)$ \\
\hline 1 & $\mathfrak{s l}_{2 n}$ & $\mathfrak{s l}_{n} \oplus \mathfrak{s l}_{n} \oplus \mathfrak{t}_{1}$ & $\mathfrak{s l}_{n}$ & $\mathbf{C}_{n}$ & 2 & $\mathbf{A}_{n-1}$ \\
2 & $\mathfrak{s p}_{2 n}$ & $\mathfrak{g l}_{n}$ & $\mathfrak{s o}_{n}$ & $\mathbf{C}_{n}$ & 1 & $\mathbf{A}_{n-1}$ \\
3 & $\mathfrak{s o}_{4 n}$ & $\mathfrak{g l}_{2 n}$ & $\mathfrak{s p}_{2 n}$ & $\mathbf{C}_{n}$ & 4 & $\mathbf{A}_{n-1}$ \\
4 & $\mathfrak{s o}_{n+2}$ & $\mathfrak{s o}_{n} \oplus \mathfrak{s o}_{2}$ & $\mathfrak{s o}_{n-1}$ & $\mathbf{C}_{2}$ & $n-2$ & $\mathbf{A}_{1}$ \\
5 & $\mathbf{E}_{7}$ & $\mathbf{E}_{6} \oplus \mathfrak{t}_{1}$ & $\mathbf{F}_{4}$ & $\mathbf{C}_{3}$ & 8 & $\mathbf{A}_{2}$ \\
\hline
\end{tabular}

Table 2. Restricted root systems associated with Jordan triads.

Proposition 6.5. For all items in Table 2, we have

$$
\operatorname{dim} \mathfrak{E}(\mathcal{J}) \geqslant \operatorname{dim} \mathcal{J}+\left(m_{\text {short }}-1\right)[r / 2],
$$

where $r$ is the rank of $\Psi(G / H)$.

Proof. Using Theorem 6.3, we identify $\mathfrak{E}(\mathcal{J})$ with the zero fiber of the quadratic covariant $\mathfrak{g}_{10} \times \mathfrak{g}_{11} \rightarrow \mathfrak{g}_{10}$ and work in the setting of Section 5 . Let $\varepsilon_{1}, \ldots, \varepsilon_{r}$ be the usual basis of $\mathfrak{X}\left(C_{11}\right) \otimes \mathbb{Q}$ such that the roots of $\Psi(G / H)$ are $\pm \varepsilon_{i} \pm \varepsilon_{j}(i \neq j)$ and $\pm 2 \varepsilon_{i}$. The roots in $\Psi\left(H / G_{00}\right)$ are $\pm\left(\varepsilon_{i}-\varepsilon_{j}\right)$. Therefore, $\mathfrak{g}_{10} \oplus \mathfrak{g}_{01}$ is the sum of root spaces corresponding to $\pm\left(\varepsilon_{i}+\varepsilon_{j}\right)$ and $\pm 2 \varepsilon_{i}$. Set

$$
\tilde{\mathfrak{c}}=\left\{x \in \mathfrak{c}_{11} \mid\left(\varepsilon_{i}+\varepsilon_{r+1-i}\right)(x)=0 \text { for } i=1,2, \ldots,\left[\frac{r+1}{2}\right]\right\} .
$$

Then $\operatorname{dim} \tilde{\mathfrak{c}}=[r / 2]$, and we have $2[r / 2]$ short roots of $\mathfrak{g}_{10} \oplus \mathfrak{g}_{01}$ vanishing on $\tilde{\mathfrak{c}}$. Moreover, if $r$ is odd, then the long roots $\pm 2 \varepsilon_{[r+1 / 2]}$ also vanish on $\tilde{\mathfrak{c}}$. Therefore,

$$
\operatorname{dim}_{\mathfrak{z} \mathfrak{g}}(\tilde{\mathfrak{c}})_{10}=\frac{1}{2} \operatorname{dim}\left(\mathfrak{z}_{\mathfrak{g}}(\tilde{\mathfrak{c}}) \cap\left(\mathfrak{g}_{10} \oplus \mathfrak{g}_{01}\right)\right)= \begin{cases}m_{\text {short }} \cdot r / 2 & \text { if } r \text { is even, } \\ m_{\text {short }} \cdot[r / 2]+1 & \text { if } r \text { is odd. }\end{cases}
$$

In both cases, this yields $\operatorname{dim} G_{00} \cdot\left(\mathfrak{z}_{\mathfrak{g}}(\tilde{\mathfrak{c}})_{10} \oplus \tilde{\mathfrak{c}}\right)=\operatorname{dim} \mathfrak{g}_{11}+\left(m_{\text {short }}-1\right)[r / 2]$.

For the Jordan algebra of all matrices (related to a Hermitian involution of $\mathfrak{s t}_{2 n}$ ), the above construction of $\tilde{\mathfrak{c}}$ gives exactly the subvariety of Example 6.4. It is plausible that the lower bound of Proposition 6.5 provides the exact value of $\operatorname{dim} \mathfrak{E}(\mathcal{J})$.

Remark 6.6. It is curious that, for all Hermitian involutions leading to Jordan triads, the restricted root system is of type $\mathbf{C}_{n}$; whereas, for all other Hermitian involutions, the restricted root system $\Psi$ is of type $\mathbf{B C}_{n}$. Namely, the symmetric pairs $\mathfrak{g l}_{n+m} \supset \mathfrak{g l}_{n} \times \mathfrak{g l}_{m} \times \mathfrak{t}_{1}(n<m)$ and $\mathfrak{s o}_{4 n+2} \supset \mathfrak{g l}_{2 n+1}$ lead to $\Psi \simeq \mathbf{B C}_{n}$; $\mathbf{E}_{6} \supset \mathbf{D}_{5} \times \mathfrak{t}_{1}$ leads to $\Psi \simeq \mathbf{B C}_{2}$.

\section{Appendix: Computations in classical Lie algebras}

Here we provide some computations related to the proof of Theorem 4.4 for nilpotent elements in classical Lie algebras. 
Let $\lambda=\left(\lambda_{1}, \ldots, \lambda_{s}\right)$ be a partition and $e \in \mathfrak{g l}_{n}$ a nilpotent element corresponding to $\lambda$, also denoted by $e \sim \lambda$. Then $\sum \lambda_{i}=n$ and

$$
\operatorname{dim}\left(\mathfrak{g l}_{n}\right)^{e}=n+2 \sum_{i<j} \min \left\{\lambda_{i}, \lambda_{j}\right\}, \quad \operatorname{dim}\left(\mathfrak{s l}_{n}\right)^{e}=\operatorname{dim}\left(\mathfrak{g l}_{n}\right)^{e}-1 .
$$

If $e$ is a nilpotent element in $\mathfrak{s o}_{n}$ or $\mathfrak{s p}_{2 n}$, with respective parity conditions on $\lambda$, then

$$
\begin{aligned}
\operatorname{dim}\left(\mathfrak{s p}_{2 n}\right)^{e} & =\frac{\operatorname{dim}\left(\mathfrak{g l}_{2 n}\right)^{e}+\#\left\{i \mid \lambda_{i} \text { is odd }\right\}}{2}, \\
\operatorname{dim}\left(\mathfrak{s o}_{n}\right)^{e} & =\frac{\operatorname{dim}\left(\mathfrak{g l}_{n}\right)^{e}-\#\left\{i \mid \lambda_{i} \text { is odd }\right\}}{2} .
\end{aligned}
$$

See [Hesselink 1976, (3.8); Kraft and Procesi 1982, 2.4]. Below, we consider several symmetric pairs with classical $\mathfrak{g}$ and check that (4-2) is satisfied for all nonzero nilpotent elements of $\mathfrak{g}_{0}$. There is no need to consider only noneven nilpotent elements in $\mathfrak{g}_{0}$, since the computations go through without this assumption.

A.1 $\left(\mathfrak{g}, \mathfrak{g}_{0}\right)=\left(\mathfrak{s l}_{n}, \mathfrak{s} \mathfrak{o}_{n}\right)$. If $e \in \mathfrak{s o}_{n}$ and $e \sim \lambda$, then using (A.1) and (A.3) yields $\operatorname{dim} \mathfrak{g}_{0}^{e}=\frac{\operatorname{dim}\left(\mathfrak{g l}_{n}\right)^{e}-\#\left\{i \mid \lambda_{i} \text { is odd }\right\}}{2}, \quad \operatorname{dim} \mathfrak{g}_{1}^{e}=\frac{\operatorname{dim}\left(\mathfrak{g l}_{n}\right)^{e}+\#\left\{i \mid \lambda_{i} \text { is odd }\right\}}{2}-1$.

Therefore, $\operatorname{dim} \mathfrak{g}_{0}^{e}-\operatorname{dim} \mathfrak{g}_{1}^{e}+(n-1)=n-\#\left\{i \mid \lambda_{i}\right.$ is odd $\}$. Here the parity condition means that each even part of $\lambda$ occurs an even number of times. Since $e \neq 0$, that is, $\lambda \neq(1, \ldots, 1)$, the minimal value is 2 , and it is attained for $\lambda=\left(3,1^{n-3}\right)$.

A.2 $\left(\mathfrak{g}, \mathfrak{g}_{0}\right)=\left(\mathfrak{s p}_{2 n}, \mathfrak{g l}_{n}\right)$. If $e \in \mathfrak{g l}_{n}$ and $e \sim \lambda$, then the partition of $e$ as an element of $\mathfrak{s p}_{2 n}$ is obtained by doubling $\lambda$, that is, each part $\lambda_{i}$ is replaced with $\left(\lambda_{i}, \lambda_{i}\right)$. Then $\operatorname{dim} \mathfrak{g}_{0}^{e}=\operatorname{dim}\left(\mathfrak{g l}_{n}\right)^{e}$ is given by (A.1), and using (A.2) yields

$$
\operatorname{dim} \mathfrak{g}_{1}^{e}=2 \sum_{i}\left[\frac{\lambda_{i}+1}{2}\right]+2 \sum_{i<j} \min \left\{\lambda_{i}, \lambda_{j}\right\}
$$

Hence

$$
\operatorname{dim} \mathfrak{g}_{0}^{e}-\operatorname{dim} \mathfrak{g}_{1}^{e}+n=2 n-2 \sum_{i}\left[\frac{\lambda_{i}+1}{2}\right]=n-\#\left\{i \mid \lambda_{i} \text { is odd }\right\} .
$$

For $e \neq 0$, the minimal value 2 is attained for $\lambda=\left(2,1^{n-2}\right)$ or $\left(3,1^{n-3}\right)$.

A.3 $\left(\mathfrak{g}, \mathfrak{g}_{0}\right)=\left(\mathfrak{s o}_{2 n}, \mathfrak{g l}_{n}\right)$. If $e \in \mathfrak{g l}_{n}$ and $e \sim \lambda$, then $\operatorname{dim} \mathfrak{g}_{0}^{e}=\operatorname{dim}\left(\mathfrak{g l}_{n}\right)^{e}$ is again given by (A.1), using this time (A.3), and we obtain

$$
\operatorname{dim} \mathfrak{g}_{1}^{e}=2 \sum_{i}\left[\frac{\lambda_{i}}{2}\right]+2 \sum_{i<j} \min \left\{\lambda_{i}, \lambda_{j}\right\}
$$


Hence the result is even better than in the previous case. Indeed, we have here $\operatorname{dim} \mathfrak{g}_{0}^{e}-\operatorname{dim} \mathfrak{g}_{1}^{e} \geqslant 0$.

A.4 $\left(\mathfrak{g}, \mathfrak{g}_{0}\right)=\left(\mathfrak{s l}_{n+m}, \mathfrak{s l}_{n} \times \mathfrak{s l}_{m} \times \mathfrak{t}_{1}\right)$. Here $n, m \geqslant 1$. A nilpotent element $e \in \mathfrak{g}_{0}$ is determined by two partitions, $e \sim(\boldsymbol{\lambda} ; \boldsymbol{\mu})=\left(\left(\lambda_{1}, \ldots, \lambda_{k}\right) ;\left(\mu_{1}, \ldots, \mu_{s}\right)\right)$. Using (A.1), we obtain

$$
\begin{aligned}
\operatorname{dim} \mathfrak{g}_{0}^{e} & =n+m-1+2 \sum_{i<j} \min \left\{\lambda_{i}, \lambda_{j}\right\}+2 \sum_{i<j} \min \left\{\mu_{i}, \mu_{j}\right\}, \\
\operatorname{dim} \mathfrak{g}_{1}^{e} & =2 \sum_{i, j} \min \left\{\lambda_{i}, \mu_{j}\right\} .
\end{aligned}
$$

Therefore,

$$
\begin{aligned}
\operatorname{dim} \mathfrak{g}_{0}^{e}- & \operatorname{dim} \mathfrak{g}_{1}^{e}+(n+m-1) \\
& =2\left(n+m-1+\sum_{i<j} \min \left\{\lambda_{i}, \lambda_{j}\right\}+\sum_{i<j} \min \left\{\mu_{i}, \mu_{j}\right\}-\sum_{i, j} \min \left\{\lambda_{i}, \mu_{j}\right\}\right) .
\end{aligned}
$$

Since $n=\sum_{i} \lambda_{i}, m=\sum_{j} \mu_{j}$, and $\sum_{i<j} \min \left\{\lambda_{i}, \lambda_{j}\right\}=\sum_{i \geqslant 2}(i-1) \lambda_{i}$, half of the right-hand side equals

$$
\mathcal{F}(\lambda ; \boldsymbol{\mu}):=\sum_{i=1}^{k} i \lambda_{i}+\sum_{j=1}^{s} j \mu_{j}-1-\sum_{i=1}^{k} \sum_{j=1}^{s} \min \left\{\lambda_{i}, \mu_{j}\right\}
$$

Arguing by induction, we prove that $\mathcal{F}(\lambda ; \boldsymbol{\mu}) \geqslant 0$ for all $\lambda$ and $\boldsymbol{\mu}$, and if $n+m \geqslant 3$, then $\mathcal{F}(\lambda ; \boldsymbol{\mu})>0$.

(1) First, $\mathcal{F}\left(1^{n} ; 1^{m}\right)=(n-m)^{2} / 2+(n+m) / 2-1$, which is positive if $(n, m) \neq(1,1)$.

(2) The inequality is easily verified, if $\lambda$ or $\boldsymbol{\mu}$ consists of only one part.

(3) Suppose that $k \geqslant 2$ and $s \geqslant 2$. Write $\lambda=\left(\lambda_{1}, \lambda^{\prime}\right)$ and $\boldsymbol{\mu}=\left(\mu_{1}, \boldsymbol{\mu}^{\prime}\right)$. Then

$$
\begin{aligned}
\mathcal{F}(\lambda ; \boldsymbol{\mu}) & =\mathcal{F}\left(\lambda^{\prime} ; \boldsymbol{\mu}^{\prime}\right)+\max \left\{\lambda_{1}, \mu_{1}\right\}+\sum_{i \geqslant 2}\left(\lambda_{i}-\min \left\{\lambda_{i}, \mu_{1}\right\}\right)+\sum_{j \geqslant 2}\left(\mu_{j}-\min \left\{\lambda_{1}, \mu_{j}\right\}\right) \\
& \geqslant \mathcal{F}\left(\lambda^{\prime} ; \boldsymbol{\mu}^{\prime}\right)+\max \left\{\lambda_{1}, \mu_{1}\right\} \geqslant \max \left\{\lambda_{1}, \mu_{1}\right\} .
\end{aligned}
$$

Here $\max \left\{\lambda_{1}, \mu_{1}\right\}$ arises as $\lambda_{1}+\mu_{1}-\min \left\{\lambda_{1}, \mu_{1}\right\}$.

We omit the computations related to the remaining classical symmetric pairs $\left(\mathfrak{s l}_{2 n}, \mathfrak{s p}_{2 n}\right),\left(\mathfrak{s p}_{2 n+2 m}, \mathfrak{s p}_{2 n} \times \mathfrak{s p}_{2 m}\right)$, and $\left(\mathfrak{s o}_{n+m}, \mathfrak{s o}_{n} \times \mathfrak{s o}_{m}\right)$.

\section{Acknowledgements}

Part of this work was done while I was visiting the Max-Planck-Institut für Mathematik (Bonn). 


\section{References}

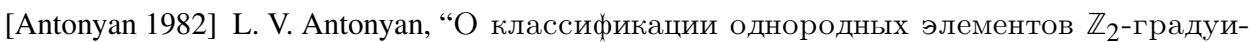
рованных полупростых алгебр Ли”, Vestnik Moskov. Univ. Ser. I Mat. Mekh. 2 (1982), 29-34. Translated as "Classification of homogeneous elements of $\mathbb{Z}_{2}$-graded semisimple Lie algebras" in Moscow Univ. Math. Bulletin, 37:2 (1982), 36-43. MR 84c:17006 Zbl 0494.17008

[Brion 2000] M. Brion, "Invariants et covariants des groupes algébriques réductifs", pp. 83-168 in Théorie des invariants et géométrie des variétés quotients (Monastir, 1996), edited by G. W. Schwarz and M. Brion, Travaux en Cours 61, Hermann, Paris, 2000. Zbl 1095.14003

[Bulois 2011] M. Bulois, "Irregular locus of the commuting variety of reductive symmetric Lie algebras and rigid pairs”, Transform. Groups 16:4 (2011), 1027-1061. MR 2012i:14061 Zbl 06031650

[Dynkin 1952] E. B. Dynkin, “Полупростые подалгебры полупростых алгебр Ли”, Mat. Sbornik (N.S.) 30(72) (1952), 349-462. Translated as "Semisimple subalgebras of semisimple Lie algebras", pp. 111-244 in Five papers on algebra and group theory by E. B. Dynkin et al., Amer. Math. Soc. Transl. (2) 6, Amer. Math. Soc., Providence, RI, 1957 (see also [Dynkin 2000, pp. 175-308]). MR 13,904c Zbl 0048.01701

[Dynkin 2000] E. B. Dynkin, Selected papers of E. B. Dynkin with commentary, edited by A. A. Yushkevich et al., Amer. Math. Soc., Providence, RI, 2000. MR 2001g:01050 Zbl 1056.01014

[Helgason 1978] S. Helgason, Differential geometry, Lie groups, and symmetric spaces, Pure and Applied Mathematics 80, Academic Press, New York, 1978. MR 80k:53081 Zbl 0451.53038

[Hesselink 1976] W. H. Hesselink, "Singularities in the nilpotent scheme of a classical group", Trans. Amer. Math. Soc. 222 (1976), 1-32. MR 55 \#2885 Zbl 0332.14017

[Kac 1980] V. G. Kac, "Some remarks on nilpotent orbits", J. Algebra 64:1 (1980), 190-213. MR 81i:17005 Zbl 0431.17007

[Kantor 1964] I. L. Kantor, "Классификация неприводимых транзитивно-дифференциальных групп”, Dokl. Akad. Nauk SSSR 158:6 (1964), 1271-1274. Translated as "Classification of irreducible transitively differential groups" in Sov. Math. Dokl. 5 (1965), 1404-1407. MR 31 \#217 Zbl 0286.17011

[Kollross 2009] A. Kollross, "Exceptional $\mathbb{Z}_{2} \times \mathbb{Z}_{2}$-symmetric spaces", Pacific J. Math. 242:1 (2009), 113-130. MR 2010j:17023 Zbl 1184.53056

[Kostant and Rallis 1971] B. Kostant and S. Rallis, "Orbits and representations associated with symmetric spaces”, Amer. J. Math. 93 (1971), 753-809. MR 47 \#399 Zbl 0224.22013

[Kraft and Procesi 1982] H. Kraft and C. Procesi, "On the geometry of conjugacy classes in classical groups”, Comment. Math. Helv. 57:4 (1982), 539-602. MR 85b:14065 Zbl 0511.14023

[Panyushev 1994a] D. I. Panyushev, "Complexity and nilpotent orbits", Manuscripta Math. 83:3-4 (1994), 223-237. MR 95e:14039 Zbl 0822.14024

[Panyushev 1994b] D. I. Panyushev, "The Jacobian modules of a representation of a Lie algebra and geometry of commuting varieties", Compositio Math. 94:2 (1994), 181-199. MR 95m:14030 Zbl 0834.17003

[Panyushev 1999] D. I. Panyushev, "On the conormal bundle of a $G$-stable subvariety", Manuscripta Math. 99:2 (1999), 185-202. MR 2000e:14081 Zbl 0961.14030

[Panyushev 2013] D. I. Panyushev, "Commuting involutions and degenerations of isotropy representations”, Transform. Groups 18:2 (2013), 507-537. MR 3055775

[Panyushev and Yakimova 2007] D. I. Panyushev and O. Yakimova, "Symmetric pairs and associated commuting varieties”, Math. Proc. Cambridge Philos. Soc. 143:2 (2007), 307-321. MR 2008k:14090 Zbl 1126.17010 
[Premet 2003] A. Premet, "Nilpotent commuting varieties of reductive Lie algebras", Invent. Math. 154:3 (2003), 653-683. MR 2004k:20090 Zbl 1068.17006

[Richardson 1979] R. W. Richardson, "Commuting varieties of semisimple Lie algebras and algebraic groups”, Compositio Math. 38:3 (1979), 311-327. MR 80c:17009 Zbl 0409.17006

[Richardson 1981] R. W. Richardson, "An application of the Serre conjecture to semisimple algebraic groups", pp. 141-151 in Algebra, Carbondale 1980: Lie algebras, group theory, and partially ordered algebraic structures (Carbondale, IL, 1980), edited by R. K. Amayo, Lecture Notes in Math. 848, Springer, Berlin, 1981. MR 83j:20047 Zbl 0457.14022

[Richardson 1982] R. W. Richardson, "On orbits of algebraic groups and Lie groups", Bull. Austral. Math. Soc. 25:1 (1982), 1-28. MR 83i:14041 Zbl 0467.14008

[Richardson 1987] R. W. Richardson, "Normality of $G$-stable subvarieties of a semisimple Lie algebra", pp. 243-264 in Algebraic groups (Utrecht, 1986), edited by A. M. Cohen et al., Lecture Notes in Math. 1271, Springer, Berlin, 1987. MR 90d:14052 Zbl 0632.14011

[Tits 1962] J. Tits, “Une classe d'algèbres de Lie en rélation avec les algèbres de Jordan”, Nederl. Akad. Wetensch. Proc. (A) 65 (1962), 530-535. MR 26 \#3753 Zbl 0104.26002

[Vasconcelos 1994] W. V. Vasconcelos, Arithmetic of blowup algebras, London Mathematical Society Lecture Note Series 195, Cambridge University Press, 1994. MR 95g:13005 Zbl 0813.13008

[Vergne 1995] M. Vergne, "Instantons et correspondance de Kostant-Sekiguchi", C. R. Acad. Sci. Paris (I) Math. 320:8 (1995), 901-906. MR 96c:22026 Zbl 0833.22010

[Vinberg 1976] Ѐ. В. Vinberg, "Группа Вейля градуированной алгебры Ли”, Izv. Akad. Nauk SSSR Ser. Mat. 40:3 (1976), 488-526. Translated as "The Weyl group of a graded Lie algebra" in Math. USSR-Izv. 10 (1976), 463-495. MR 55 \#3175 Zbl 0363.20035

[Vinberg 1979] Ѐ. В. Vinberg, “Классификация однородных нильпотентных элементов полупростой градуированной алгебры Ли”, Trudy Sem. Vektor. Tenzor. Anal. 19 (1979), 155-177. Translated as "Classification of homogeneous nilpotent elements of a semisimple graded Lie algebra” in Selecta Math. Sov. 6 (1987), 15-35. MR 80k:17006 Zbl 0431.17006

[Vinberg 2005] È. B. Vinberg, "Short $\mathrm{SO}_{3}$-structures on simple Lie algebras and associated quasielliptic planes", pp. 243-270 in Lie groups and invariant theory, edited by È. B. Vinberg, Amer. Math. Soc. Transl. (2) 213, Amer. Math. Soc., Providence, RI, 2005. MR 2006d:17008 Zbl 1081.17009

Communicated by J. Toby Stafford

Received 2012-09-19 Accepted 2013-01-24

panyushev@iitp.ru

Dobrushin Mathematics Laboratory, Institute for Information Transmission Problems, Russian Academy of Sciences, Bolshoy Karetny per. 19, Moscow, 127994, Russia 


\section{Algebra \& Number Theory}

msp.org/ant

\section{EDITORS}

MANAGING EDITOR

Bjorn Poonen

Massachusetts Institute of Technology

Cambridge, USA

\author{
EDITORIAL BOARD CHAIR \\ David Eisenbud \\ University of California \\ Berkeley, USA
}

\section{BOARD OF EDITORS}

Georgia Benkart

Dave Benson

Richard E. Borcherds

John H. Coates

J-L. Colliot-Thélène

Brian D. Conrad

Hélène Esnault

Hubert Flenner

Edward Frenkel

Andrew Granville

Joseph Gubeladze

Ehud Hrushovski

Craig Huneke

Mikhail Kapranov

Yujiro Kawamata

János Kollár

Yuri Manin

Barry Mazur

Philippe Michel
University of Wisconsin, Madison, USA

University of Aberdeen, Scotland

University of California, Berkeley, USA

University of Cambridge, UK

CNRS, Université Paris-Sud, France

University of Michigan, USA

Freie Universität Berlin, Germany

Ruhr-Universität, Germany

University of California, Berkeley, USA

Université de Montréal, Canada

San Francisco State University, USA

Hebrew University, Israel

University of Virginia, USA

Yale University, USA

University of Tokyo, Japan

Princeton University, USA

Northwestern University, USA

Harvard University, USA

École Polytechnique Fédérale de Lausanne
Susan Montgomery

Shigefumi Mori

Raman Parimala

Jonathan Pila

Victor Reiner

Karl Rubin

Peter Sarnak

Joseph H. Silverman

Michael Singer

Vasudevan Srinivas

J. Toby Stafford

Bernd Sturmfels

Richard Taylor

Ravi Vakil

Michel van den Bergh

Marie-France Vignéras

Kei-Ichi Watanabe

Efim Zelmanov
University of Southern California, USA

RIMS, Kyoto University, Japan

Emory University, USA

University of Oxford, UK

University of Minnesota, USA

University of California, Irvine, USA

Princeton University, USA

Brown University, USA

North Carolina State University, USA

Tata Inst. of Fund. Research, India

University of Michigan, USA

University of California, Berkeley, USA

Harvard University, USA

Stanford University, USA

Hasselt University, Belgium

Université Paris VII, France

Nihon University, Japan

University of California, San Diego, USA

\section{PRODUCTION}

production@msp.org

Silvio Levy, Scientific Editor

See inside back cover or msp.org/ant for submission instructions.

The subscription price for 2013 is US \$200/year for the electronic version, and \$350/year ( $\$ 40$, if shipping outside the US) for print and electronic. Subscriptions, requests for back issues and changes of subscribers address should be sent to MSP.

Algebra \& Number Theory (ISSN 1944-7833 electronic, 1937-0652 printed) at Mathematical Sciences Publishers, 798 Evans Hall \#3840, c/o University of California, Berkeley, CA 94720-3840 is published continuously online. Periodical rate postage paid at Berkeley, CA 94704, and additional mailing offices.

ANT peer review and production are managed by EditFLOW ${ }^{\circledR}$ from Mathematical Sciences Publishers.

\section{PUBLISHED BY}

- mathematical sciences publishers

nonprofit scientific publishing

http://msp.org/

(C) 2013 Mathematical Sciences Publishers 


\section{Algebra \& Number Theory}

Volume $7 \quad$ No. $6 \quad 2013$

On the discrete logarithm problem in elliptic curves II

Claus Diem

Identifying Frobenius elements in Galois groups

1325

TIM DOKCHITSER and VLADIMIR DOKCHITSER

Weak approximation for cubic hypersurfaces of large dimension

1353

MIKE SWARBRICK JONES

The Picard crossed module of a braided tensor category

1365

ALEXEI DAVYDOV and DMITRI NIKSHYCH

A Gross-Zagier formula for quaternion algebras over totally real fields

1405

EYAL Z. GOREN and KRISTIN E. LAUTER

Counting rational points over number fields on a singular cubic surface

CHRISTOPHER FREI

On the ample cone of a rational surface with an anticanonical cycle

ROBERT FRIEDMAN

Commuting involutions of Lie algebras, commuting varieties, and simple Jordan algebras

DMITRI I. PANYUSHEV 